\author{
环糊精聚合物及其生物医学应用的研究进展 \\ 郄淑燕 ${ }^{\dagger,}, a$ 郝莹 ${ }^{\dagger, b} \quad$ 刘宗建 $^{a}$ 王锦*, ${ }^{*}$ 席家宁*, $a$ \\ ( ${ }^{a}$ 首都医科大学附属北京康复医院 北京 100044) \\ ( ${ }^{b}$ 中国科学院苏州纳米技术与纳米仿生研究所 苏州 215123)
}

\begin{abstract}
摘要 环糊精是一类由吡喃葡萄糖单元构成的大环分子, 能够通过包结作用与多种小分子、金属离子以及聚合物形成 主一客体包结物, 从而实现对客体分子理化性能的调控, 被广泛用于制药、食品、化学、色谱、催化、生物技术、农业、 化妆品、卫生、医学、纺织和环境等领域. 然而, 环糊精分子在众多应用领域不易操控，通过化学交联制备环糊精聚合 物, 形成以环糊精为主体的聚合物, 则有望实现环糊精与交联基团的 “集成” 和 “协同” 作用, 不但能有效解决环糊精 分子的操控问题，而且能够赋予环糊精单分子所不具有的独特功能和理化性质，因此环糊精聚合物的设计合成及其应 用吸引了广泛的研究兴趣. 本综述介绍了环糊精聚合物领域的研究近况, 对环糊精聚合物的种类按结构与性质进行了 分类, 系统介绍了各类环糊精聚合物的制备方法, 重点介绍了近五年环糊精聚合物取得的新突破, 并详细论述了各类 环糊精聚合物在生物医药中的应用和其他领域的代表性应用, 最后对环糊精聚合物作了简要总结和展望.
\end{abstract}

关键词＼cjkstart环糊精; 聚合物; 化学交联; 药物载体; 超分子自组装

\title{
Advances in Cyclodextrin Polymers and Their Applications in Biomedicine
}

\author{
Qie, Shuyan ${ }^{\dagger, a}$ Hao, Ying ${ }^{\dagger, b} \quad$ Liu, Zongjian ${ }^{a} \quad$ Wang, Jin ${ }^{*, b} \quad \mathrm{Xi}$, Jianing*,a \\ ( ${ }^{a}$ Beijing Rehabilitation Hospital of Capital Medical University, Beijing 100044) \\ ( ${ }^{b}$ Suzhou Institute of Nano-Tech and Nano-Bionics, Chinese Academy of Sciences, Suzhou 215123)
}

\begin{abstract}
Cyclodextrins (CDs) are a family of macrocyclic oligosaccharides composed of $\alpha$-1,4-linked $D$-glucopyranose units. The most commonly used CDs are $\alpha-, \beta$-, and $\gamma$-CD, which consist of 6,7 , and $8 D$-glucose units, respectively. They possess a relative hydrophobic inner cavity and a hydrophilic outer surface and can form inclusion complexes with various small molecules, metal ions and polymers, tailoring the physicochemical property of the guests, and thus have been widely used in the fields of pharmacy, food, chemistry, chromatography, catalysis, biotechnology, agriculture, cosmetics, hygiene, medicine, textiles and the environment, etc. However, it is difficult to manipulate the native CDs in some specific applications, they are crystallized solid and soluble in water but insoluble in most organic solvents. CD polymers (CDPs), such as crosslinked CDs or CD based hydrogels with various crosslinkers by chemical reactions, and CD based supramolecular polymers formed by physical interactions, can achieve the integration effect and synergy effect of CDs, crosslinkers and guest polymers, not only possessing the inclusion capacity of CDs, but also endowing CDs with other properties introduced by crosslinkers. The CDPs can be easily manipulated and they exhibit unique features that native CDs are lack of. Hence, the design, synthesis and applications of CDPs have attracted broad interests in recent years. This review focuses on the recent progress in CDPs, and different types of CDPs are identified and classified based on the structures and functions, namely CD based polyrotaxane, grafted CDs, crosslinked CDs and linear CDs, etc. Besides, the synthetic methodologies of CDPs are highlighted. Particular attention is paid to the breakthrough on the CDPs in the past five years, and their applications in biomedicine, such as drug delivery, gene delivery, target delivery, controlled release and cell imaging are discussed in detail. The typical applications in other fields such as absorption, environment remediation, thermal insulation, catalysis and slid-ring gels are also discussed in brief. Finally, the review provides brief summary and prospect of CDPs.

Keywords cyclodextrin; polymer; chemical crosslinking; drug carrier; supramolecular self-assembly
\end{abstract}

\section{1 引言}

环糊精(cyclodextrin，简称 $\mathrm{CD})$ 是一类由吡喃葡萄 糖单元通过 $\alpha-1,4-$ 糖苷键链接而成的大环分子，具有疏
水空腔和亲水外壁的中空圆台状立体结构, 能够通过包 结作用(encapsulation)与多种小分子和聚合物形成主-客 体(host-guest)包结物(inclusion complexes), 从而实现对 客体分子物理和化学性能的调控, 被广泛用于制药、食

\footnotetext{
* E-mail: jwang2014@sinano.ac.cn; xijn888@sina.com

$\uparrow$ These authors contributed equally to this work.

Received January 8, 2020; published February 26, 2020.

Project supported by the National Natural Science Foundation of China (Nos. 91963124, 51773225, 51903246).

项目受国家自然科学基金(Nos. 91963124, 51773225, 51903246)资助.
} 
品、化学、色谱、催化、生物技术、农业、化妆品、卫 生、医学、纺织和环境等领域 ${ }^{[1 \sim 8]}$. 最常见的 $\mathrm{CD}$ 由 6 、 7 或 8 个吡喃葡萄糖单元构成, 分别称为 $\alpha-、 \beta$-和 $\gamma-\mathrm{CD}$, 并分别具有 $18 、 21$ 和 24 个羟基, 空腔直径分别约 0.49 、 0.65 和 $0.8 \mathrm{~nm}$, 厚度皆为 $0.8 \mathrm{~nm}$ 左右. 纯 $\mathrm{CD}$ 都具有高 度结晶结构, $\mathrm{CD}$ 分子之间相互堆叠, 空腔通常被相邻 $\mathrm{CD}$ 阻隔, 因此 $\mathrm{CD}$ 的固态包结能力较弱, 客体分子难以 扩散至 $\mathrm{CD}$ 晶体内部. 将 $\mathrm{CD}$ 充分溶解在二甲基甲酰胺 (DMF)中, 再通过加温可实现 $\alpha$-和 $\gamma-\mathrm{CD}$ 的有序排列和 管道结晶的形成, 使 CD 空腔面与面堆叠形成单分散亚 纳米孔道结构, 将 $\mathrm{CD}$ 分子的空腔完全展露出来 ${ }^{[9]}$, 虽 然其固-液吸附能力有一定的提高, 但与理论计算值仍 然相差较远, 主要原因在于表层 $\mathrm{CD}$ 的空腔包覆客体分 子之后, 缺乏往内运动的驱动力. 通过喷雾干燥法可抑 制 $\mathrm{CD}$ 结晶并获得无定形 $\mathrm{CD}$ 颗粒，提高了固态下 $\mathrm{CD}$ 的吸附能力 ${ }^{[10,11]}$, 但 $\mathrm{CD}$ 的无定形结构极不稳定, 在湿 度或温度较高情况下都能自发结晶. 此外, 纯 $\mathrm{CD}$ 分子 在水等溶剂中溶解度高, 不便于收集使用. 因此, 如何 实现 $\mathrm{CD}$ 包结能力的充分展现、如何将 $\mathrm{CD}$ 制备成便于 操控的材料, 一直是学术界和产业界关注的核心问题.

以 $\mathrm{CD}$ 为主体成分, 通过化学交联等方式制备得到 的一类聚合物, 分子骨架中具有众多 $\mathrm{CD}$ 基元, 因此被 称为环糊精聚合物 (cyclodextrin polymers, 简称 $\mathrm{CDPs})^{[12 \sim 14]}$. CDPs 分为可溶和不溶两大类, 能维持环糊 精固有的大环结构和功能差基, 又使其具有高分子或交 联网络的性质，表现出高度的 “集成” 和 “协同” 效应， 不但解决前述 $\mathrm{CD}$ 分子的问题, 充分开发 $\mathrm{CD}$ 分子的潜 力, 而且赋予其单分子所不具有的独特功能和理化性 质. 早于 20 世纪 60 年代已有文献报道 $\mathrm{CDPs}^{[15,16]}$, 目前 研究得最为广泛的是环氧氯丙烷(Epichlorohydrin, 简称 $\mathrm{EPI}$ )交联的 $\beta$ - $\mathrm{CD}$ 环糊精聚合物, 通常仅用于环境中污 染物的处理 ${ }^{[12,13]}$. 自 1990 年发现 CD 与线形聚合物自组 装后, 研究人员制备了一系列生物相容性好、生物可降 解的 CDP — 超分子聚轮烷 (Polyrotaxane, 简称 $\mathrm{PR})^{[17 \sim 23]}$. 鉴于 $\mathrm{CD}$ 优异的生物相容性、可降解性以及 与多种有机、无机大小分子的包结作用能力, 使得 CDP 在生物医药领域中的应用大放光彩 ${ }^{[4,14]}$.

本综述系统概述了 CDPs 的种类及其制备方法, 特 别是近 5 年来其在合成方法、新交联剂、新聚合物体系 与形态等方面取得的突破性进展. 归纳总结了环糊精聚 合物的应用现状, 特别是在生物医药领域应用的最新研 究成果. 最后总体展望了环糊精聚合物在功能化设计、 新结构和生物医药中的应用前景和发展趋势.

\section{2 环糊精聚合物的种类}

图 1 概括了 CDPs 的几种类型, 根据 $\mathrm{CD}$ 分子的特 征, 可分为: (1)环糊精聚轮烷(图 1a). CD 分子穿在线形 聚合物链上，聚合物两端由大体积基团或聚合物封端防
止 $\mathrm{CD}$ 分子脱离, 主要特征为 $\mathrm{CD}$ 与聚合物和封端基团 无化学作用, $\mathrm{CD}$ 分子可在聚合物链上滑移和旋转, $\mathrm{CD}$ 上的羟基完全保留，可进一步修饰，但 $\mathrm{CD}$ 空腔被聚合 物占据无法实现进一步包结; (2)环糊精接枝聚合物(图 1b). CD 分子通过化学键接枝到聚合物侧基或枝化高分 子端基上, 主要特征为 $\mathrm{CD}$ 运动能力强, 空腔开放、能 够包结, 但 CD 在体系中的含量相对较少; (3)环糊精交 联聚合物(图 1c). 以 $\mathrm{CD}$ 分子为主要成分, 通过交联剂 进行化学交联形成聚合物网络或凝胶, 主要特征为 $\mathrm{CD}$ 数量众多, 空腔开放能够包结, 能够进一步改性修饰, 但凝胶网络不能溶解; (4) CD 线形交联聚合物(图 1d). $\mathrm{CD}$ 分子为共单体, 化学交联成线形聚合物, 特征为 $\mathrm{CD}$ 为主链的一部分, 空腔保留, 但合成难度大、极易交联 形成 $\mathrm{CD}$ 交联聚合物, 或以 $\mathrm{CD}$ 为功能基团, 在 $\mathrm{CD}$ 上接 枝数量可控的聚合物链制备得到星型聚合物, 这类 CDP 更多地体现接枝聚合物的性能. 在 CDP 的制备过 程中选择哪种 $\mathrm{CD}$ 主要取决于应用场合, 已经确定具体 客体分子，则选择能与其进行包结的 $\mathrm{CD}$, 例如制备环 糊精聚轮烷选用的客体为聚乙二醇(poly(ethylene oxide)，简称 PEO)时，只能选用 $\alpha$-或 $\gamma-\mathrm{CD}^{[1,2]}$; 当客体分子 不确定时，如环糊精交联聚合物用于吸附时，则从成本 考虑的较多, 众多研究工作选择成本相对较低的 $\beta-\mathrm{CD}^{[12,13]}$.

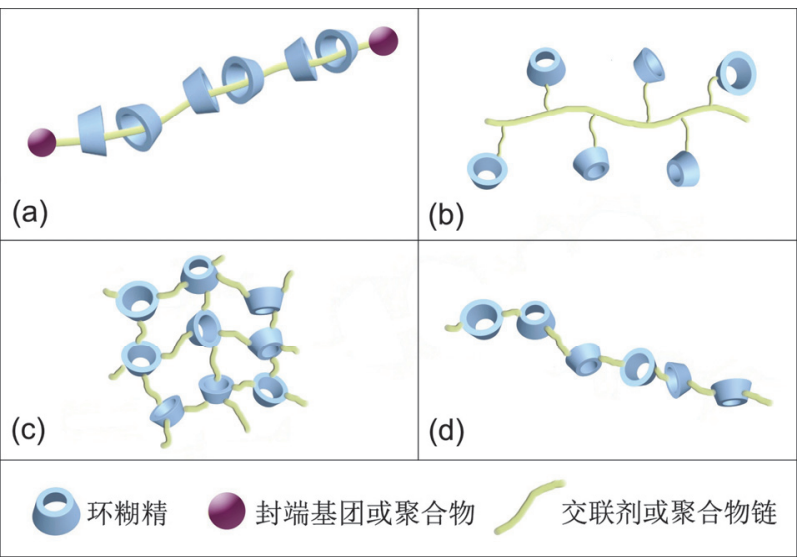

图 1 环糊精聚合物的结构示意图: (a)环糊精聚轮烷; (b)环糊精接枝 聚合物; (c)环糊精交联聚合物; (d) 环糊精线形交联聚合物

Figure 1 Types of cyclodextrin polymers: (a) polyrotaxane; (b) grafted CD polymers; (c) crosslinked CD polymers; and (d) crosslinked linear $\mathrm{CD}$ polymers

\section{1 环糊精聚轮烷(CD-PR)}

CD-PR 的制备方法主要有三种 ${ }^{[24]}$, 如图 2 所示, 分 别为: (1) Threading approach, 即 CD 首先通过自组装与 聚合物形成聚准轮烷包结物, 随后封端制备得到 PR, 是最常用的方法; (2) Slippage approach, 即 CD 穿越大体 积基团，直接与哑铃状分子形成 PR; (3) Inclusion polymerization approach, 即 $\mathrm{CD}$ 分子与聚合物单体包结, 随后原位聚合形成 $P R$, 这种方法在 $\mathrm{CD}$ 与聚合物自组装 发现前应用得较为普遍 ${ }^{[24]}$. 从结构上看, CD-PR 主要由 
三部分构成, $\mathrm{CD}$ 主体分子、客体聚合物链和封端基团. 其中 $\alpha-、 \beta$-和 $\gamma-\mathrm{CD}$ 都能作为主体分子, 而能用于客体的 聚合物也较多, 一般取决于 $\mathrm{CD}$ 的种类, 迄今用于 PR 的 客体聚合物有 PEO、聚丙二醇(poly(propylene oxide), 简 称 PPO)、PEO-PPO 嵌段共聚物(Pluronic 系列等)、聚四 氢呋喃(Polytetrahydrofuran, 简称 PTHF)、聚 $\varepsilon$-己内酯 (Poly( $\varepsilon$-caprolactone), 简称 $\varepsilon$-PCL)、聚乳酸(Polylactic acid, 简称 PLA)、聚异丁烯(Polyisobutene, 简称 PIB)、 聚二甲基硅氧烷(Polydimethylsiloxane, 简称 PDMS)、聚 ( $N$-异丙基丙烯酰胺)(Poly( $N$-isopropyl acrylamide), 简称 PNIPAAm)等, 其中 PEO 和 PPO 是采用最多的客体高分 子[25 32].

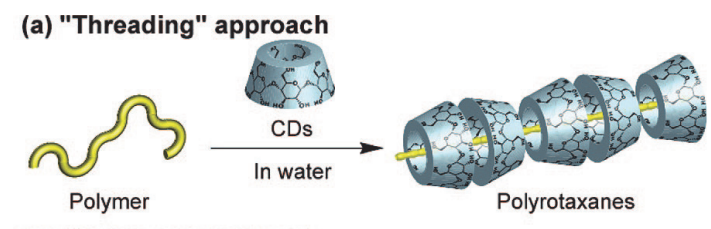

(b) "Slippage" approach

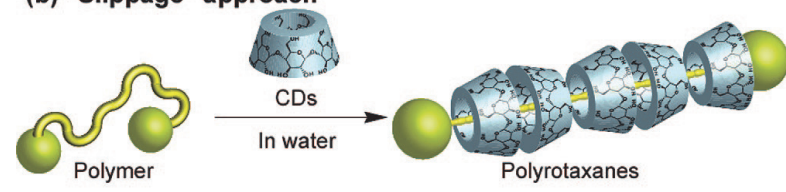

(c) "Inclusion polymerization" approach

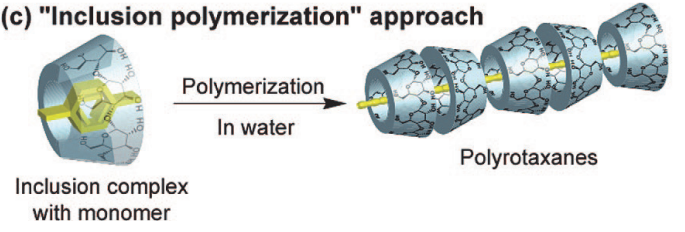

图 2 环糊精聚轮烷的制备方法: (a)穿线法; (b)穿越法; (c)包结物原位 聚合法 ${ }^{[24]}$

Figure 2 Synthetic approaches for CD based PRs: (a) "Threading" approach; (b) "Slippage" approach; and (c) "Inclusion polymerization" approach. Reprinted with permission from ref. ${ }^{[24]}$. Copyright $\odot 2009$ American Chemical Society.
主-客体分子的选择主要取决于二者之间的包结性 能，而封端基团往往决定 PR 体系的降解性、溶解性、 以及大尺度的组装行为, 因此从应用角度出发, 封端基 团的调控也是极其重要的环节. 图 3a 列举了常用的封 端基团小分子 ${ }^{[24]}$ ，这类封端基团一般是在 DMF、二甲基 亚砜(DMSO)等溶剂中通过偶联反应键合到聚准轮烷的 两端, 与之相应地, 聚准轮烷主链的两端也需要经过官 能团功能化, 最常用的是端氨基, 此外, 端着基和端羧 基也采用过. 采用小分子封端基团需在 DMF、DMSO 等溶剂中进行，会造成 $\mathrm{CD}$ 分子的滑脱，所得的 PR一般 不溶于水、四氢呋喃(THF)等溶剂中. 此外, $\mathrm{CD}$ 自身也 被用作封端基团 ${ }^{[33,34]}$.

为了解决以上 PR 面临的问题，原子转移自由基聚 合(atom transfer radical polymerization, 简称 ATRP)于 2008 年首次被引入到 PR 的制备中 ${ }^{[35 ~ 37]}$, 即制备聚准轮 烷引发剂, 再采用 ATRP 法在聚准轮烷两端原位聚合乙 烯基单体，得到聚合物封端的三嵌段聚轮烷共聚物. 如 图 3b 所示, 本方法可选用的封端单体种类众多，且具有 传统方法无法比拟的优势: (1)反应可以在水相、室温条 件下进行; (2)一锅法聚合; (3)可选用的乙烯基单体众多, 结构和分子量可控; (4)引入聚合物封端, 使 PR 溶于水 并自组装成胶束、囊泡等结构，也可进一步熔融加工、 电纺丝等; (5)可在两端的聚合物链上进行 PR 的改性从 而保持 $\mathrm{CD}$ 不受影响. 由此制备了一系列生物相容性 好、具有刺激响应性和优异力学性能的 $\mathrm{PR}^{[38 \sim 46]}$, 在药 物控制释放、组织工程支架中具有潜在的应用价值. 以 PNIPAAm 聚合物封端的聚轮烷为例, Feng 等 ${ }^{[38,41]}$ 采用 Pluronic F127 为客体聚合物，经双端基溴甲基化后，在 环糊精饱和水溶液中自组装形成聚准轮烷, 随后加入 NIPAAm 单体，原位进行 ATRP 聚合封端得到具有温度

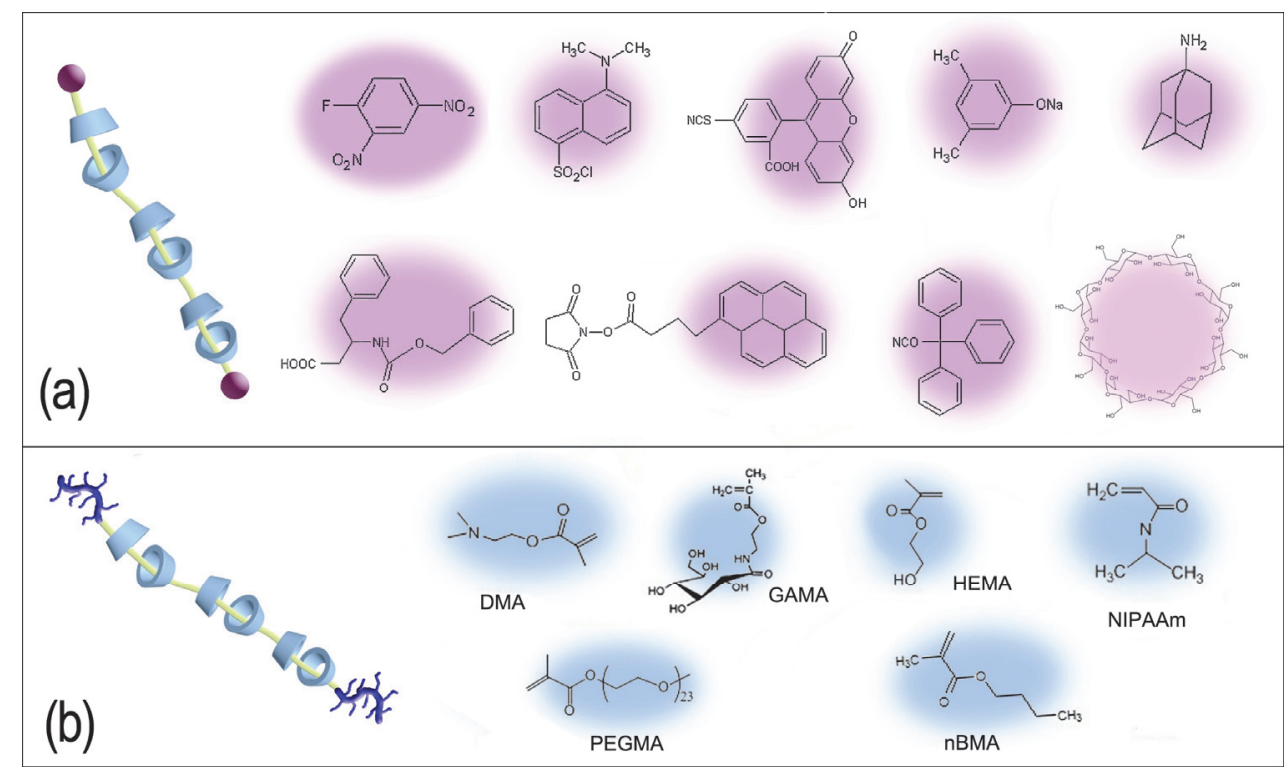

图 3 环糊精聚轮烷封端基团的种类: (a)小分子封端基; (b)聚合物封端基

Figure 3 End-capping groups of CD based PRs: (a) small molecule end-capping groups; and (b) polymeric end-capping groups. 
和溶剂双重刺激响应的聚轮烷. 由于在水相中聚合, 包 结后的 $\mathrm{CD}$ 不易掉脱, 因此能够通过物质的量配比实现 聚轮烷中 CD 数量的精确控制, 所得的聚轮烷也具有较 好的水溶性, 封端聚合物 PNIPAAm 的分子量也可精确 控制.

最近, 多种新类型 CD-PR 不断被合成并报道出来, 如通过 $\alpha-\mathrm{CD}$ 与 PEO 在水中自组装形成超分子水凝胶, 再通过超临界二氧化碳干燥, 制备得到具有二维片层骨 架结构的超分子聚轮烷气凝胶, 该气凝胶具有 $45 \mathrm{~m}^{2} / \mathrm{g}$ 的比表面积, 比表面积大小可通过 CD 的种类、 PEO 分 子量大小、 $\mathrm{CD}$ 与 $\mathrm{PEO}$ 物质的量配比进行调控, 所得气 凝胶微块体材料, 能够支撑自身重量 1000 倍以上的重 物, 具有管道结晶和 PEO 结晶双晶区, 将气凝胶的隔热 性能和相变储能功能有机融合一体 ${ }^{[47]}$; 以 PNIPAAm 封 端的温度与溶剂双重刺激响应的聚轮烷为单体, 通过 $\mathrm{CD}$ 上的差基进行化学交联制备得到有机凝胶, 再通过 超临界干燥得到温度刺激响应聚轮烷气凝胶, 此类聚轮 烷为块体, 具有丰富的开孔结构, 孔隙率达到 $90 \%$ 以上, 密度最低仅 $0.1 \mathrm{~g} / \mathrm{cm}^{3}$, 杨氏模量达到 $74.7 \mathrm{MPa}$, 压缩 $80 \%$ 以上也不破碎, 表现出优异的韧性. 从其扫描电子 显微镜图可以看出, 该气凝胶骨架为纳米颗粒, 与前者 的二维纳米片层完全不同. X 射线衍射(XRD)结果表明, 此气凝胶为无规结构, 相比结晶性材料, 韧性有一定的 提高. 由于聚轮烷两端的封端基团为 PNIPAAm, 其结 构能够完好地保存下来, 因此实现了超分子本体材料在 固态下的刺激响应性, 即高温时, 此气凝胶表现为疏水 性, 接触角大于 $90^{\circ}$, 当温度低于 $32{ }^{\circ} \mathrm{C}$ 时, 表现为亲水 性, 接触角小于 $90^{\circ}{ }^{[48]}$; 此外, 通过客体聚合物端基的 修饰, 使形成的聚准轮烷纳米片相互排斥, 制备得到独 立的聚准轮烷二维纳米片层, 在药物载体与输送等方面 具有潜在的应用价值 ${ }^{[49,50]}$; 以光响应化学键链接的封端 基团制备聚轮烷, 得到可逆的光刺激降解 PR, 如图 4 所 示 $^{[51]}$, 由硫代苯甲酸酯封端的 PR, 在紫外光照下封端 基团断裂, $\mathrm{CD}$ 分子脱离, $\mathrm{PR}$ 解体, 在黑暗条件下封端基 团又键合, 得到 $\mathrm{CD}$ 数量较少的 PR. 以上工作是该领域 近来取得的部分进展, 结构和性能上都有别于传统的 PR 体系, 极大地丰富和促进了 PR 的发展和应用.

\section{2 环糊精接枝聚合物(grafted-CDP)}

$\mathrm{CD}$ 接枝聚合物从结构上主要分为两种: 一种为图 $1 \mathrm{~b}$ 所示的侧链接枝型, 另一种为星型聚合物或支化聚 合物端链上的接枝. 除此之外, $\mathrm{CD}$ 也被广泛用于固相界 面的接枝修饰，不在本综述所述的 CDP 范畴内 ${ }^{[52,53]}$.

侧链接枝型 CDP 主要包括将 CD 分子通过化学键 接枝到合成高分子活性侧基上或生物大分子侧基上，合 成高分子类型有聚(甲基乙烯基醚-马来酸酐)共聚物 ${ }^{[54]}$ 、 聚丙烯酰胺(polyacrylamide) ${ }^{[55]}$ 、聚丙烯酸(Poly(acrylic acid)) 及其嵌段共聚物 ${ }^{[56]}$ 等; 天然高分子主要包括壳聚

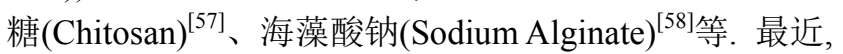

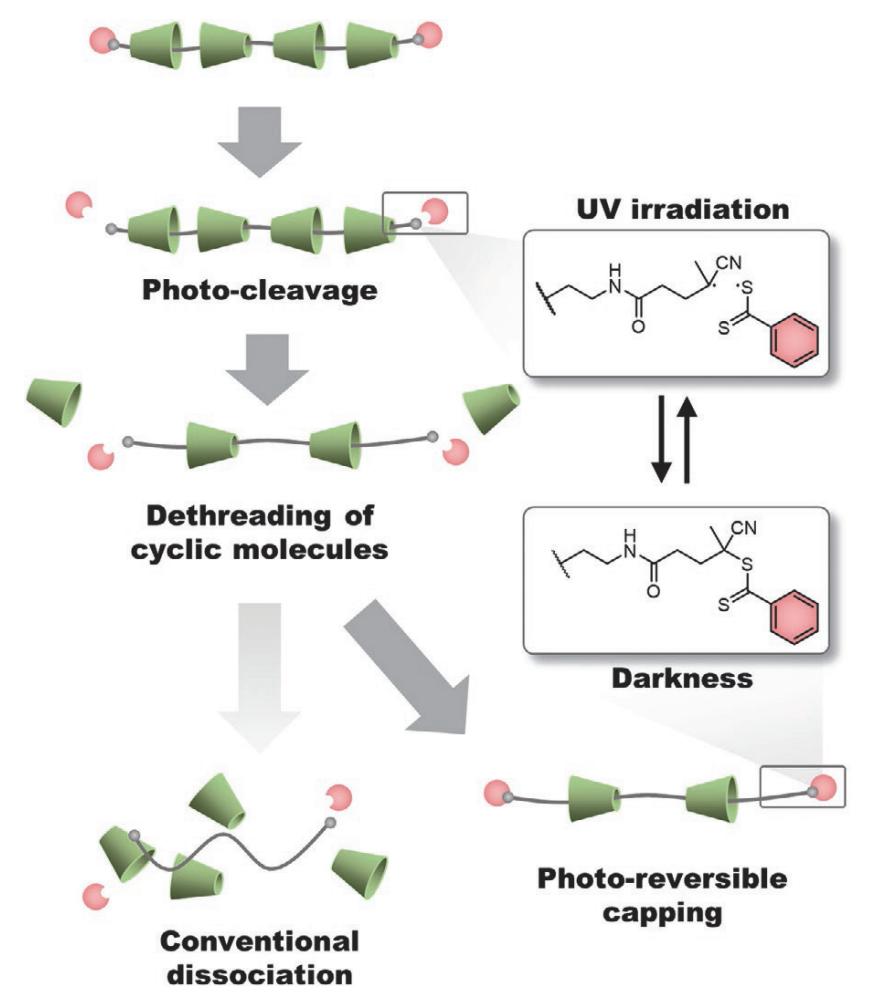

图 4 光驱动可解体的 $\mathrm{CD}$ 聚轮烷结构示意图 ${ }^{[50]}$

Figure 4 Photo-reversible dissociation process of CD based PRs. Reprinted with permission from ref. ${ }^{[50]}$. Copyright $\bigcirc 2019$ Wiley-VCH Verlag $\mathrm{GmbH} \& \mathrm{KGaA}$, Weinheim.

以氧化石墨烯(Graphene Oxide，简称 GO)为基底，合成 了 $\mathrm{CD}$ 接枝的 GO-CDP, 将线形的 $\mathrm{CD}$ 接枝聚合物扩展 到二维纳米片状接枝 $\mathrm{CDP}^{[59,60]}$. 例如 $\mathrm{Liu}$ 等 ${ }^{[60]}$ 采用 Hummers 法制备了大尺寸的 GO 纳米片，随后进行超声 得到 50 200 nm 大小、1 2 $\mathrm{nm}$ 厚的小尺寸 $\mathrm{GO}$, 最后 在 1-ethyl-3-[3-dimethylaminopropyl]carbodiimide (EDC)/ $N$-hydroxysulfosuccinimide (NHSS)催化体系下将单乙二 胺基环糊精接枝到 GO 上得到 GO-CDP. 此接枝聚合物 作为主体，与肽和偶氮苯修饰的肿瘤靶向蛋白自组装形 成更高级别的纳米聚集体.

星型 CDP 中 CD 主要位于聚合物链端, 如在超支化 聚硅氧烷表面修饰 CD 分子, 提高其苂光效率和生物相 容性 ${ }^{[61]}$; 在磁性纳米颗粒表面修饰 CD 分子得到树枝状 $\mathrm{CDP}$, 可提高纳米颗粒的选择性吸附能力(图 5) ${ }^{[62,63]}$; 在 磁性颗粒/聚吡咯纳米颗粒表面接枝 $\mathrm{CD}$, 实现阿霉素 $(\mathrm{DOX})$ 的定向输送和释放 ${ }^{[64]}$. 以上接枝 $\mathrm{CDP}$ 体系都无 一例外利用了 $\mathrm{CD}$ 的自组装功能, 主要用于构建更高层 级的组装和特异性识别, 提高目标分子的负载能力、吸 附能力、靶向功能等.

\section{3 环糊精交联聚合物(crosslinked CDP)}

$\mathrm{CD}$ 的交联聚合物是研究地最为久远的一类

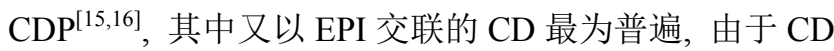
分子高度交联，与接枝 CDP 相比, CD 的空腔虽然也都 完好地保留下来, 但其运动受到限制, 因此通常只用于 


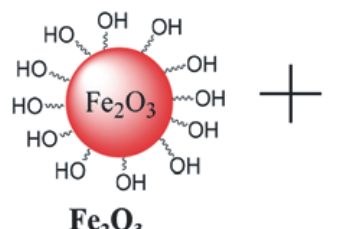<smiles>NCCCS(=O)(=O)O</smiles>
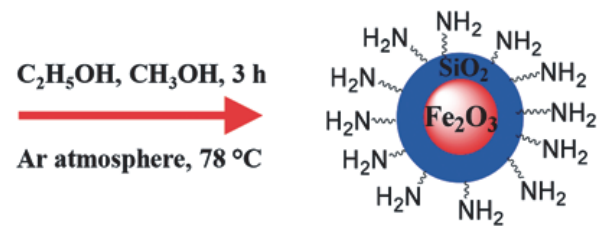

APTES

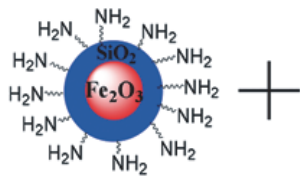

APTES@ $\mathrm{Fe}_{2} \mathrm{O}_{3}$

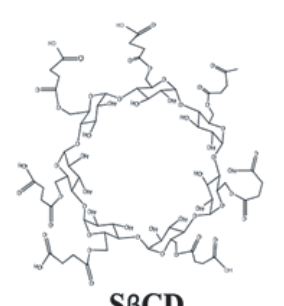

S $\beta C D$

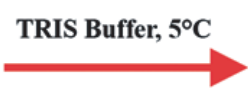

NHS / EDC
APTES@ $\mathrm{Fe}_{2} \mathrm{O}_{3}$

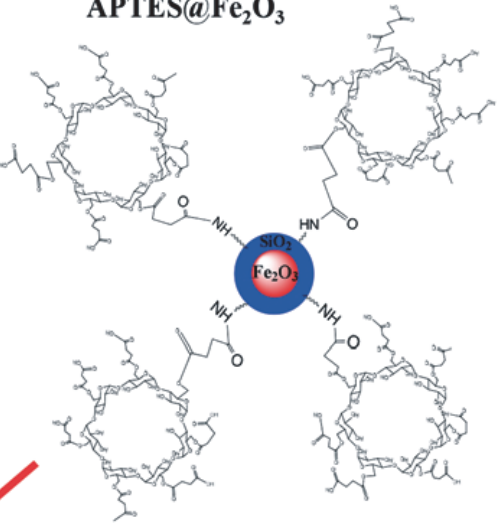

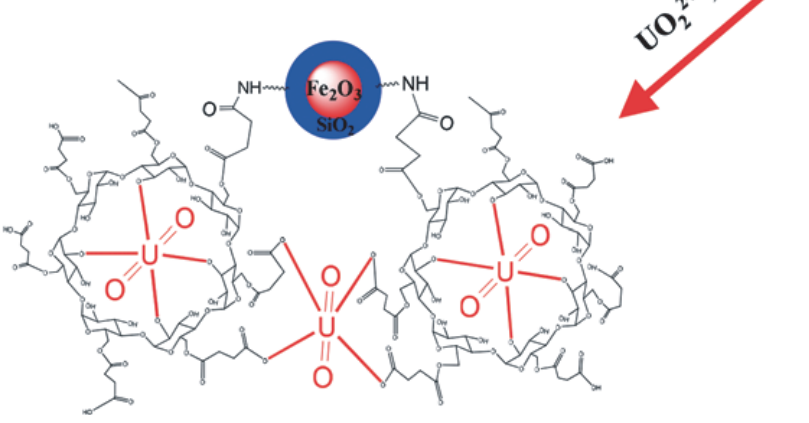

SBCD-APTES@ $@ \mathrm{Fe}_{2} \mathrm{O}_{3}$

图 5 环糊精接枝聚合物的制备: 磁性纳米颗粒表面修饰环糊精分子[63]

Figure 5 Synthesis of CD-grafted polymers: magnetic nanoparticles modified with CDs. Reprinted with permission from ref. ${ }^{[63]}$. Copyright $\odot 2018$ The Royal Society of Chemistry.

小分子的吸附, 不具有聚轮烷和接枝聚合物的自组装功 能. 关于 EPI 交联 CDP 已有文献进行了详细论述 ${ }^{[2,13]}$, 本综述重点介绍近 5 年在 $\mathrm{CD}$ 交联聚合物领域取得的新 突破, 包括结构的调控、材料维度的设计, 以及其在生 物医药中的应用.

由 EPI 交联的 CD 聚合物比表面积极低(约 $1 \sim 10$ $\mathrm{m}^{2} / \mathrm{g}$ ), 一定程度上影响了吸附质的扩散过程(吸附速率) 和吸附量. 除了 EPI, 还有其他众多试剂用来交联环糊 精 ${ }^{[12,13]}$, 主要包括各类二异氧酸酯(Diisocyanate)、酰化 聚乙二醇(Acylated poly(ethylene glycol))、已二酰氯 (Adipoyl chloride)、柠檬酸(Citric Acid)、乙二醇缩水甘 油 醚 (ethyleneglycol diglycidylether) 、戊二醛 (Glutaraldehyde)、聚丙烯酸(Poly(acrylic acid))、葵二酰 氯(Sebacoyl Chloride)、琥珀酸䣶(Succinic Anhydride)等, 上述交联剂制备的 CDP 比表面积低、无纳米孔结构. 最 近, 疏水性的刚性小分子四氟对苯二腈 (Tetrafluoroterephthalonitrile, 简称 TFTPN) 被用于交联 $\beta$-环糊精, 反应于 $80{ }^{\circ} \mathrm{C}$ 碳酸钾的 $\mathrm{THF}$ 悬浮液中进行, 所得 CDP 为黄色胶状沉淀物. 通过氮气等温吸附测量, 此环糊精聚合物具有极高的比表面积, 最高达 $263 \mathrm{~m}^{2} / \mathrm{g}$, 孔径主要分布在 $1.8 \sim 3.5 \mathrm{~nm}$, 对双酚 $\mathrm{A}$ 等有机小分子
具有吸附量大、吸附速度快等特点(图 6) ${ }^{[65]}$. 采用 TFTPN 为硬交联剂、EPI 为软交联剂, 二者以一定比例共混, 制 备得到的环糊精聚合物具有丰富的孔结构, 二氧化碳等 温吸附计算得到其比表面积达 $103 \mathrm{~m}^{2} / \mathrm{g}$, 相同条件下无 TFTPN 的 EPI 环糊精聚合物的比表面积仅 $1.2 \mathrm{~m}^{2} / \mathrm{g}$. 多 梯度的孔结构和大比表面积显著提高了该环糊精聚合 物对双酚 A、间羟基联苯、乙炔雌二醇的吸附 ${ }^{[66]}$.

此外, 上述介绍的环糊精聚合物, 无论是聚轮烷 $\mathrm{CDP}$ 、接枝 $\mathrm{CDP}$ 、以及交联 $\mathrm{CDP}$ 等. 干燥后均为固体 粉末. 最近, 中国科学院苏州纳米所研究人员分别以柔 性的六亚甲基二异氧酸酯(hexamethylene diisocyanate, 简称 HDI) 和刚性的三苯异氰酸酯甲烷(triphenylmethane-4,4',4"-triisocyanate, 简称 TTI)为交联剂，制备基于 $\beta$-环糊精的交联聚合物凝胶, 该反应在 $80{ }^{\circ} \mathrm{C}$ 的 DMF 中 进行, 不添加其他任何催化剂, 通过交联度和单体浓度 控制得到凝胶，最后通过超临界干燥技术，得到了具有 优异力学性能的块体环糊精聚合物- $\mathrm{CD}$ 气凝胶, 该 块体材料杨氏模量高达 $166 \mathrm{MPa}$, 密度仅为 $0.73 \mathrm{~g} / \mathrm{cm}^{3}$. 氮气吸附测得比表面积达到 $237 \mathrm{~m}^{2} / \mathrm{g}$, 孔容达 1.02 $\mathrm{cm}^{3} / \mathrm{g}$, 且具有 $46 \mathrm{~m}^{2} / \mathrm{g}$ 的微孔面积. 如图 7 所示, 这种环 糊精聚合物又称为环糊精气凝胶, 具有分子空腔、微孔、 

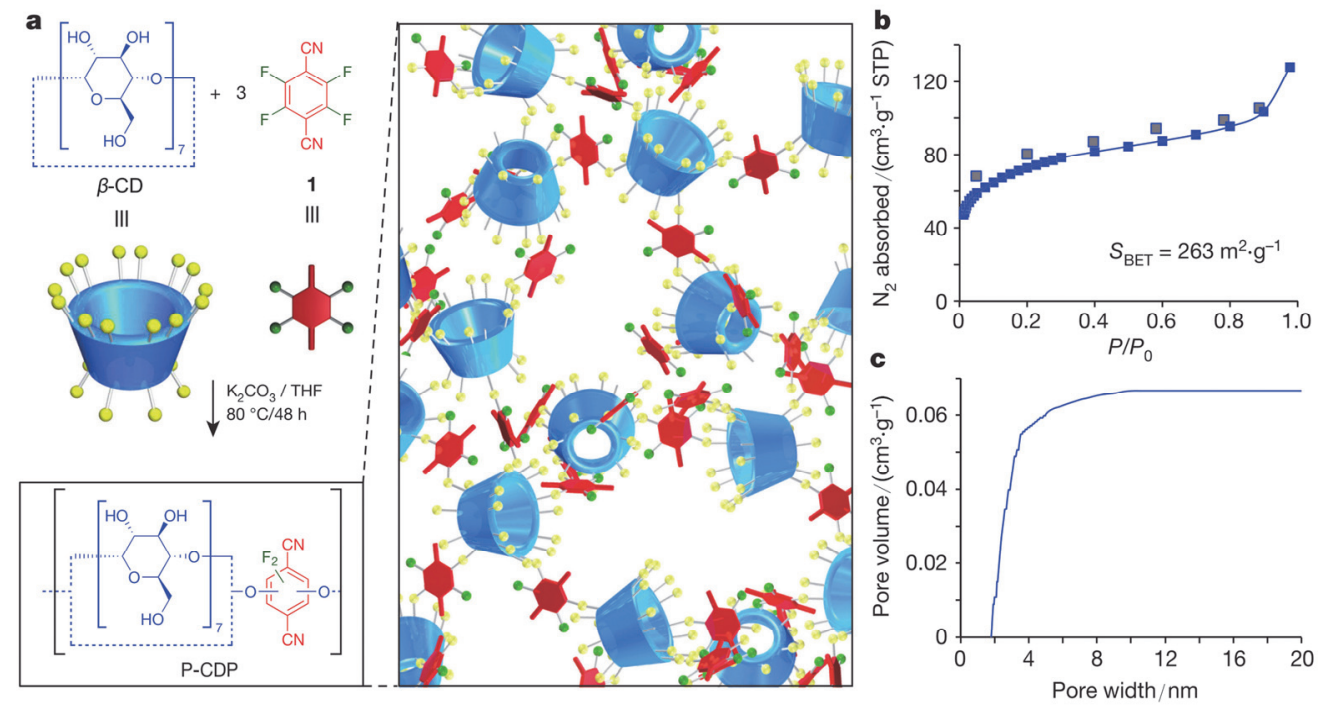

图 6 环糊精交联聚合物反应示意图、等温吸附曲线及孔径分布 ${ }^{[65]}$

Figure 6 Synthesis of crosslinked CD polymer, its adsorption isotherm and distribution of pore diameter. Reprinted with permission from ref. ${ }^{[65]}$. Copyright $\odot 2016$ Macmillan Publishers Limited.

介孔和大孔，对有机分子吸附能力强，在环境修复方面 具有潜在的应用价值 ${ }^{[67]}$. Wooley 等 ${ }^{[68]}$ 采用氨基功能化 的 $\beta$-环糊精衍生物为单体, 以均苯二甲酸酐为交联剂, 通过交联和化学脱水等步骤, 最后超临界干燥得到环糊 精一聚酰亚胺聚合物气凝胶, 该环糊精聚合物也具有多 级孔结构, 比表面积达 $155 \mathrm{~m}^{2} / \mathrm{g}$, 但无力学性能的报道.

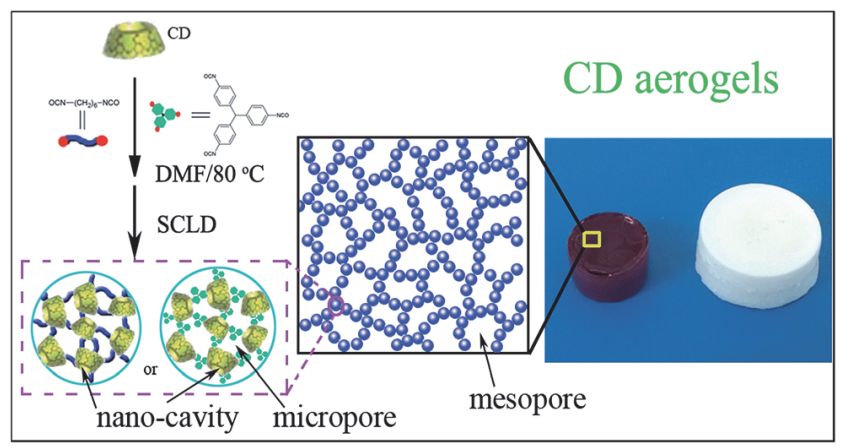

图 7 环糊精气凝胶的制备路线及实物照片 ${ }^{[67]}$

Figure 7 Synthetic approach of CD aerogels and their photo images. Reprinted with permission from ref. ${ }^{[67]}$. Copyright $₫ 2017$ The Royal Society of Chemistry.

Uyama 和 Harada 等 ${ }^{[69]}$ 采用单氯三嗪修饰的 $\beta$-环糊 精衍生物 (MCT- $\beta$-CD) 为单体, 聚乙烯亚胺 (Polyethyleneimine, 简称 PEI) 为交联剂, 不通过超临界 流体干燥也得到了环糊精交联块体材料. 将 MCT- $\beta$-CD 与 PEI 在去离子水中混合, 随后分别在 4、6、8、10、 25 和 $50{ }^{\circ} \mathrm{C}$ 反应 $72 \mathrm{~h}$, 得到一系列白色水凝胶, 将水凝 胶分别用水、乙醇和正己烷置换, 最后在室温条件下干 燥得到多孔的环糊精聚合物块体. 不同温度下反应得到 的环糊精聚合物块体密度都在 $0.2 \sim 0.25 \mathrm{~g} / \mathrm{cm}^{3}$ 之间, 但 低温(低于 $10{ }^{\circ} \mathrm{C}$ )下得到的块体都具有 $400 \mathrm{~m}^{2} / \mathrm{g}$ 以上的 比表面积, 杨氏模量最高达 $26.5 \mathrm{MPa}$. 由此可见, 交联
CDP 已经在结构(比表面积)以及三维宏观形态上(本体 高强度)都于近 5 年取得迅速的发展和突破。

\section{4 环糊精线形或星型聚合物(linear or star CDP)}

相比前三种 CDP, 线形 CDP 相对较少, 主要在于 $\mathrm{CD}$ 上的羟基数量过多，反应活性相近，因此容易形成 交联 CDP 而非线形 CDP. 其中 $\mathrm{CD}$ 二聚体是研究地最早 的一种, 如图 8 所示, Ritter 等 ${ }^{[70]}$ 合成了环糊精二聚体 $\mathbf{1}$, 以其为超分子交联剂, 可与金刚烷修饰的聚合物 $\mathbf{2}$ 自组 装形成超分子水凝胶 3. 这种超分子凝胶具有可逆的透 明度, 转变温度范围窄, 且能通过改变 1 与 $\mathbf{2}$ 的比例进 行调控. 由于其良好的亲水性和结构稳定性, 在生物医 药领域具有潜在的应用价值.

以 $\mathrm{CD}$ 为活性功能基团制备的 $\mathrm{CD}$ 星型聚合物近年 来获得广泛关注: 如以 $\beta$ - $\mathrm{CD}$ 为引发剂, 首先通过开环 聚合得到 $\beta$ - $\mathrm{CD}$ 接枝的多臂聚己内酯( $\beta$-CD-g-PCL), 进 一步将 PCL 末端羟基改性，采用 ATRP 法接枝聚甲基丙 烯酸二甲氨基乙酯(Poly(2-(dimethylamino)ethylmethacrylate, 简称 PDMAEMA), 得到一种具有良好生物相 容性 的双亲性 (Amphiphilic) $\beta$-CD- $g$-(PCL- $b$ PDMAEMA)星型聚合物 ${ }^{[71]}$. 以 HDI 扩链的聚四氢呋喃 聚氨酯为臂, $\alpha-\mathrm{CD}$ 为核, Maiti 等 ${ }^{[72]}$ 合成了生物相容性好 的 $\mathrm{CD}$-聚氨酯星型聚合物并研究了其抗癌药物的负载 和释放性能. Tilloy 等 ${ }^{[73]}$ 通过酯化反应在 $\beta$ - $\mathrm{CD}$ 上接枝上 数量可控的线形或环化油酸 (oleic acid), 极大提高了 $\beta$-CD 的水中溶解度 $(50 \sim 480 \mathrm{~g} / \mathrm{L})$ 及其表面活性. 以全 甲基化 $\beta$-CD 为核, Bonnet 等 ${ }^{[74]}$ 通过酰胺化反应制备了 各类脂肪酸接枝的多臂聚合物，此聚合物能够形成直径 约 $120 \mathrm{~nm}$ 的纳米颗粒, 在 $4{ }^{\circ} \mathrm{C}$ 能够长期稳定存在, 经 $\mathrm{MD}$ 模拟计算得到此 $\mathrm{CD}$ 多臂聚合物对阿扎那韦 (Atazanavir)具有优异的负载能力. 

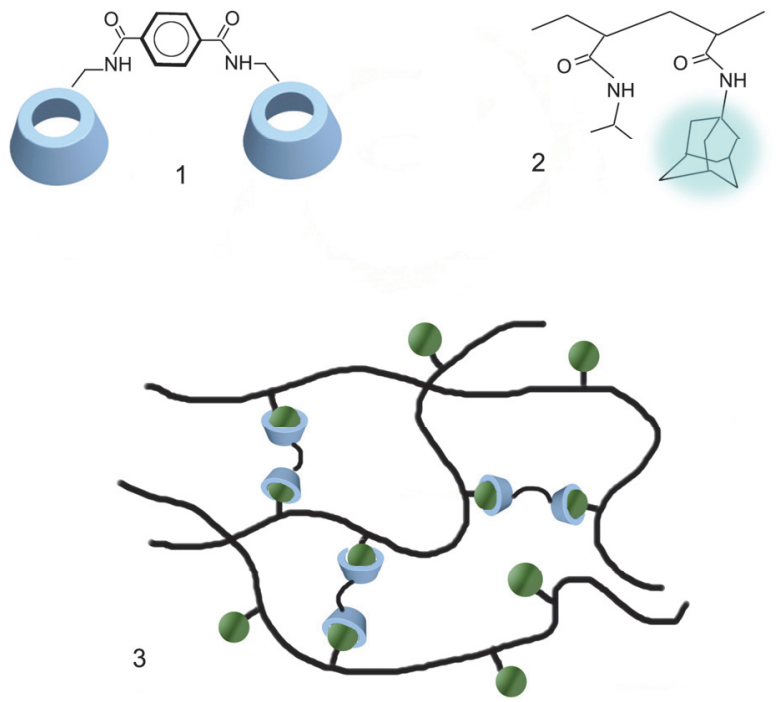

图 8 环糊精二聚体(1), 金刚烷修饰的聚合物(2)及以二聚体为交联剂 形成的超分子水凝胶(3)结构示意图

Figure 8 Structure of CD dimmer (1), adamantane modified polymer (2), and supramolecular hydrogels (3) formed by $\mathbf{1}$ and $\mathbf{2}$.

\section{3 环糊精聚合在生物医学中的应用}

$\mathrm{CDP}$ 在生物医学中具有巨大的应用价值 ${ }^{[75]}$, 主要 有赖于 CDP 的生物相容性、可降解性以及对药物分子 的高负载率和可控释放性能等, 但不同的 CDP 种类, 其 优势稍有差别, 因此本文将结合 CDP 的种类和结构特 征，重点介绍其最新进展，阐述其发挥的结构优势.

对于 $\mathrm{CD}$ 聚轮烷而言, 其主要特点和优势在于 $\mathrm{CD}$ 分子在聚合物链上的滑动和转动, 同时 $\mathrm{CD}$ 的羟基能够
完全保留，可进一步修饰实现特定功能，利用此特点研 究人员提出自适应识别或自洽识别的概念，即通过在 $\mathrm{CD}$ 上修饰抗原或配体，再利用 $\mathrm{CD}$ 的滑动和转动，可实 现高效率的自适应识别. 而传统的侧基化学键链接的配 体无法在聚合物链上滑移和转动, 长短也固定, 不具有 类似 PR 的自洽识别能力. 如图 9 所示, Yui 等 ${ }^{[76]}$ 将一种 细胞黏附短肽 (Arg-Gly-Asp, 简称 RGD)接枝在聚轮烷 的 $\mathrm{CD}$ 上，作为对比，同时合成了化学键链接的 RGD 聚 合物(图 9 中绿色线条), 研究结果表明, 在 RGD 修饰的 $\mathrm{PR}$ 中, 由于 $\mathrm{CD}$ 的运动, 能够增加 $\mathrm{RGD}$ 短肽与细胞膜 表面整合蛋白的接触频率，显著加快了早期的特异性识 别速度.

Shi 等 ${ }^{[77]}$ 在基于 $\alpha-\mathrm{CD}$ 的 PR 上同时接枝具有抗肿瘤 效应的维生素 $\mathrm{E}$ 聚乙二醇 1000 琥珀酸酯(TPGS)和 10羟基喜树碱(HCPT), 得到具有抗肿瘤活性的 CDP (PR-TPGS-HCPT). 体外实验表明, PR-TPGS-HCPT 的 抗肿瘤药物负载量达 $7.1 \%$, 在酯酶存在下能加速 HCPT 的释放，而小鼠体内实验表明，该 CDP 聚合物具有抑制 肿瘤生长、延长荷瘤小鼠的生存时间功能, 效果比单独 使用 HCPT 更佳. Yui 等 ${ }^{[78]}$ 合成了磺化 PR, 可固定成纤 维细胞生长因子(Fibroblast Growth Factor, 简称 FGF), 实现体外人间充质干细胞(Human Mesenchymal Stem Cells，简称hMSCs)的成骨分化，他们进一步发现，在移 动能力高的 PR 上, hMSCs 的黏附性差, 失去成骨分化 能力, 但在固定 FGF 的高移动性 PR 上, 成功实现了 hMSC 的分化，而在移动性能低的 PR 上，完全没有分 化. 此研究结果进一步表明 CD 中聚合物链上的移动对 特异性识别、药物释放和细胞分化有重要的促进作用.

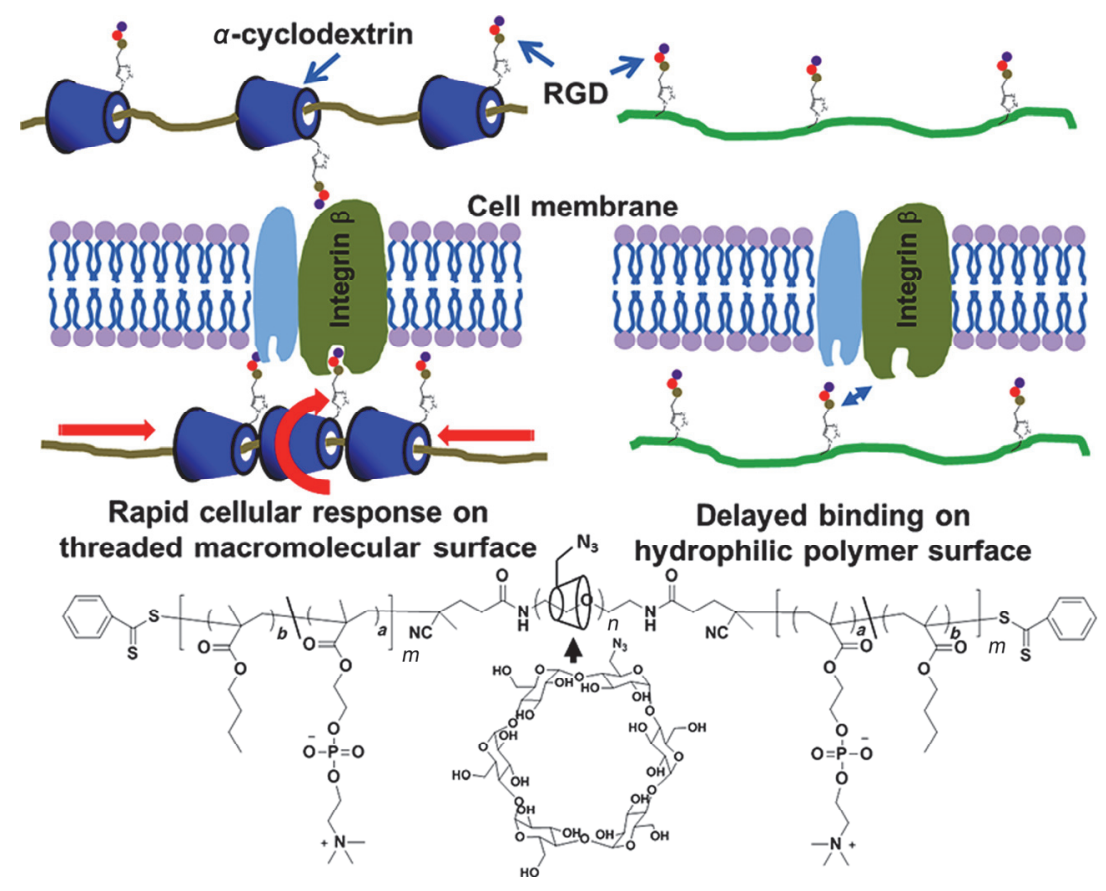

图 9 环糊精聚轮烷自适应识别、传统化学侧基聚合物示意图 ${ }^{[76]}$

Figure 9 Schematic illustration of adaptive recognition CD based PRs and traditional polymer with chemical modified side groups. Reprinted with permission from ref. ${ }^{[7]}$. Copyright $\subseteq 2013$ American Chemical Society. 
对 PR 中的 CD 进行高分子聚合物修饰, 不仅能提 高 PR 的水溶性, 而且能够有效提高药物的负载量及其 细胞相容性等 ${ }^{[34,79 ~ 82]}$. 例如 Jiang 等 ${ }^{[11]}$ 采用 ATRP 法对 PR 中的 CD 进行改性, 分别接枝上聚甲基丙烯酸-2-羟 乙酯(PHEMA)和正丁酯保护的聚甲基丙烯酸甜菜碱 $(\mathrm{PCB}-t \mathrm{Bu})$, 制备得到水溶性 PR. 其中 PHEMA 多臂聚 合物能与抗癌药物紫杉醇(PTX)结合, 负载量达 $6.6 \%$, 外层的 PCB 链段则具有优异的亲水性, 血液循环实验 表明该体系具有非常优越的缓释能力. 此外, 他们还对 PR 同时进行亲水化改性和苂光标记, 提高了 PR 的细胞 相容性, 能够通过胞吞方式被细胞摄取 ${ }^{[82]}$.

与 PR 不同，接枝型 CDPs 主要利用 $\mathrm{CD}$ 空腔的包覆 性能和自组装能力, 可实现载药、可控释放、细胞显影 增强等能力. 如图 10 所示, Liu 等 ${ }^{[60]}$ 合成了氧化石墨烯 表面接枝的 CDP (GO-CD), 利用 CD 的主体包结作用, 实现对目标客体分子的包结, 从而进一步实现功能纳米 组装体的构筑. 为此, 他们又合成了聚赖氨酸、线粒体 靶向肽、偶氮苯、以及 PEO 修饰的转铁蛋白(TPM-Azo) 并作为客体分子, GO-CD 与 TPM-Azo 通过 CD 的自组 装形成纳米组装颗粒, 对线粒体肿瘤细胞具有非常高的 靶向作用，通过光控可显著抑制肿瘤细胞的生长.

Yan 等 ${ }^{[61]}$ 合成的超支化硅氧烷-CD 接枝聚合物具有 很好的苂光增强效应, 并尝试了细胞显影应用和载药应 用. 在 $2 \mathrm{mg} / \mathrm{mL}$ 的环糊精接枝聚合物溶液中孵化 $24 \mathrm{~h}$ $\left(37{ }^{\circ} \mathrm{C}\right)$, 小鼠的成骨细胞在激光共聚焦显微镜下发蓝
光, 具有很好的显影作用. 进一步地, 他们研究了此接 枝聚合物对布洛芬的负载和释放效果，负载量达到 160 $\mathrm{mg} / \mathrm{g}$, 在弱酸性 $(\mathrm{pH}$ 6.4)条件下释放率达到 $78 \%$, 而在 中性(pH 7.4)条件下的释放率仅为 35\%(图 11).

交联型 CDP 一般难以溶解，因此主要被用于环境 水处理当中, 然而通过合成条件控制 CDP 交联微凝胶 的形成, 除了作为药物载体, 还可直接用于动脉粥样硬 化的治疗. 如图 12 所示, Park 等 ${ }^{[83]}$ 合成了尺寸为 $10 \mathrm{~nm}$ 左右的 $\mathrm{CD}$ 交联聚合物, 与纯 $\mathrm{CD}$ 相比, CDP 具有更加优 异的药代动力学和斑块靶向功效, 此外, 这种 CDP 不会 造成细胞质膜破裂，可降低 CD 的细胞毒性和溶血性. 在小鼠试验中, 皮下注射 CDP (1 g/kg) 能够有效抑制动 脉籿样硬化斑块的生长, 且在高达 $8 \mathrm{~g} / \mathrm{kg}$ 的剂量下无毒 副作用. 这些结果表明 CDP 能够有效克服纯 CD 在动脉 籿样硬化中面临的问题.

线形或星型 CDP 与接枝型 CDP 类似, 可在溶液中 自组装形成纳米颗粒, 对药物具有一定的负载作用, 可 用于药物控释应用，但与接枝 CDP 又明显不同：接枝聚 合物中 $\mathrm{CD}$ 的数量较多, $\mathrm{CD}$ 的包结作用起到的效果明 显, 而线形或星型 CDP 中 CD 含量较少, 其功能和性能 往往取决于聚合物臂的种类、分子量以及臂的数量. 如 图 13 所示, Zhong 等 ${ }^{[84]}$ 合成了 $\mathrm{CD}$ 星型聚合物, 其中 CD 的次级面被甲基化，初级面通过-SH 作用接枝上两亲性 聚合物, 该 CDP 能够进一步组装成内部疏水、外部亲水 的核-壳结构纳米胶束，尺寸在 $50 \sim 70 \mathrm{~nm}$ 之间，对

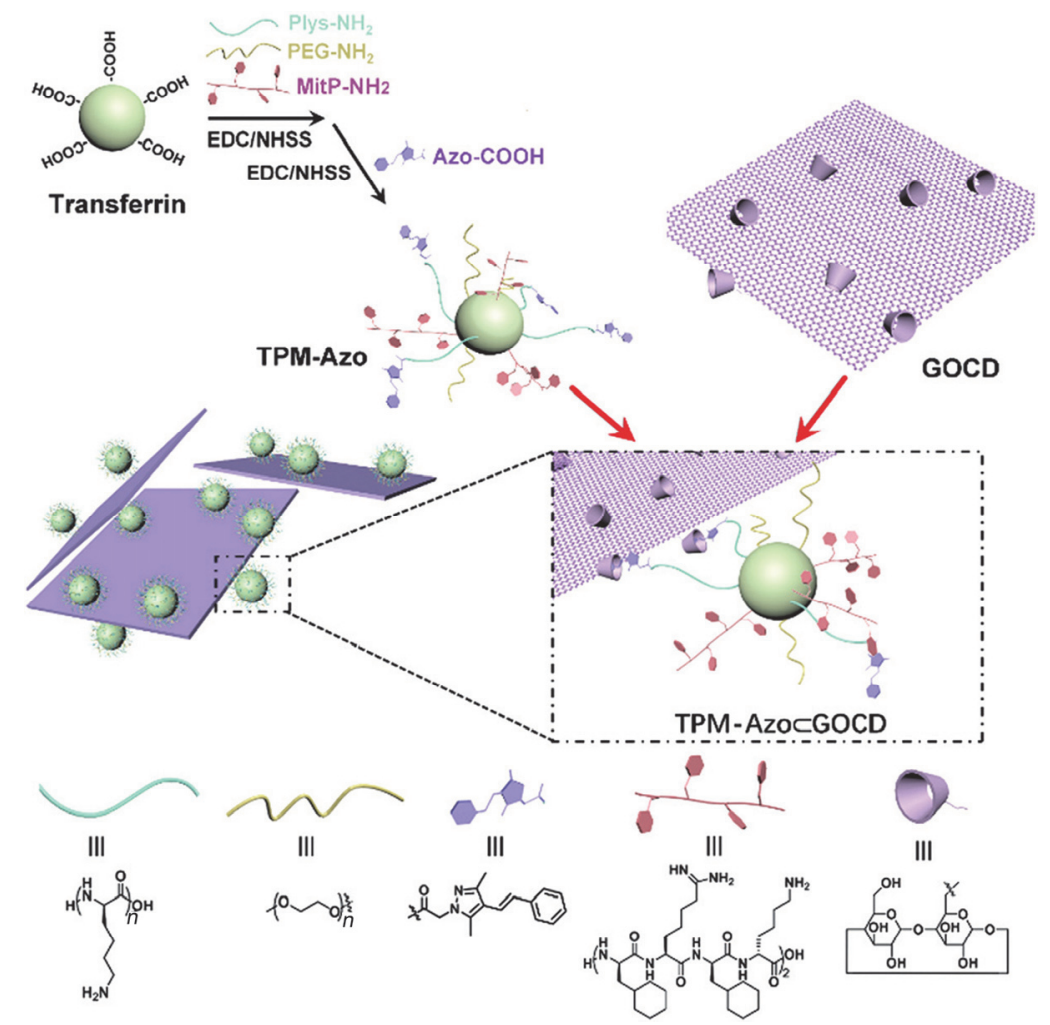

图 10 TPM-Azo 客体分子、GOCD 主体分子以及 TPM-Azo/GOCD 形成的超分子纳米组装体的合成 ${ }^{[60]}$

Figure 10 Synthesis of the TPM-Azo guest, the GOCD host, and the TPM-Azo/GOCD host-guest supramolecular nanoassemblies. Reprinted with permission from ref. ${ }^{[60]}$. Copyright $(2019$ The Royal Society of Chemistry. 


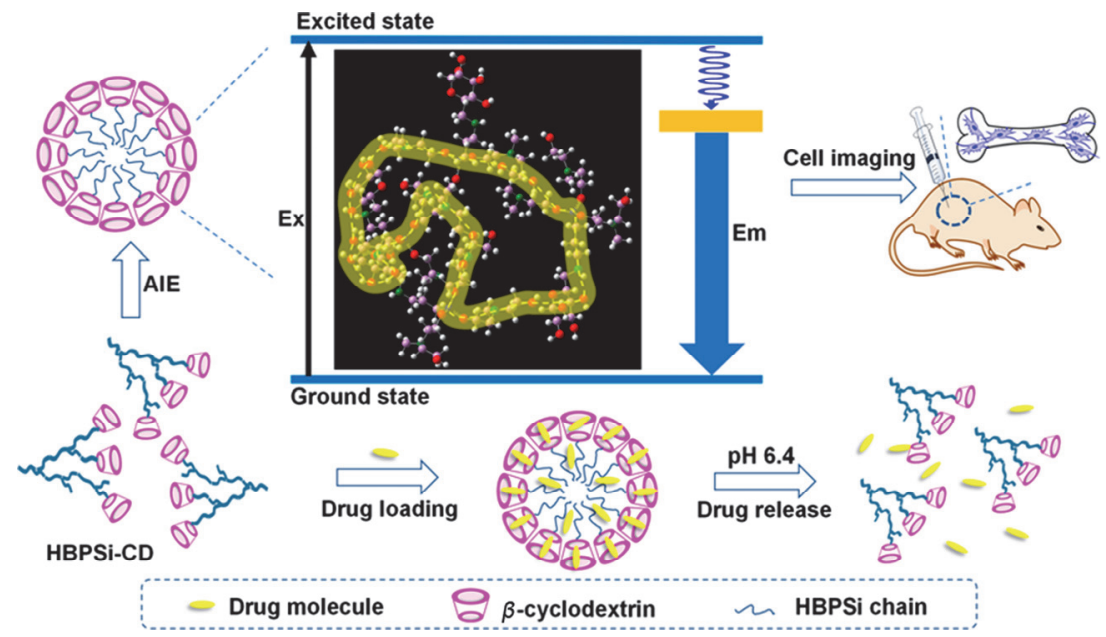

图 11 高苂光性能环糊精接枝聚合物的细胞显影及载药应用 ${ }^{[61]}$

Figure 11 High fluorescent grafted CDP for cell imaging and drug delivery. Reprinted with permission from ref. ${ }^{[61]}$. Copyright@2019 American Chemical Society.
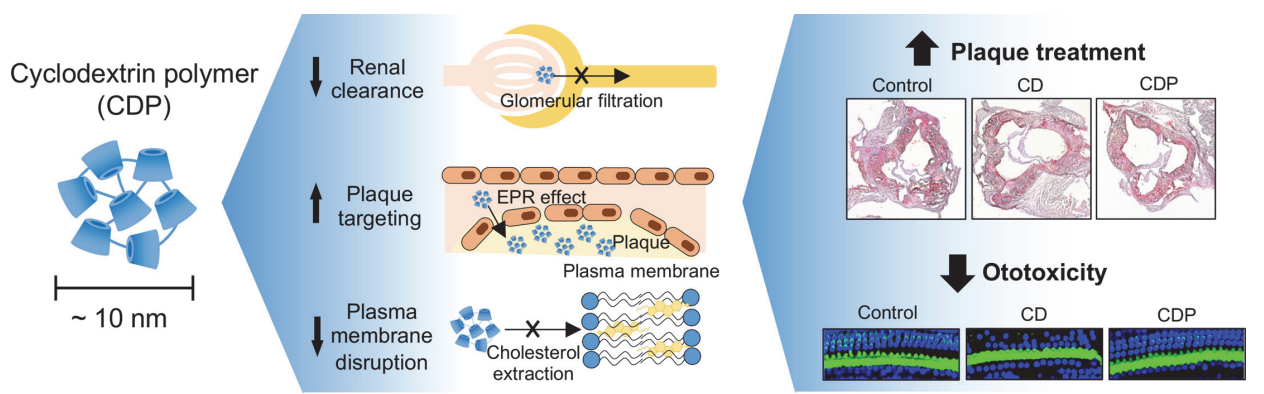

图 12 环糊精交联聚合物在动脉弹样硬化中的有效和安全应用 ${ }^{[83]}$

Figure $12 \mathrm{CDP}$ for effect and safe treatment of atherosclerosis. Reprinted with permission from ref. ${ }^{[83]}$. Copyright@2020 Elsevier B.V.

A
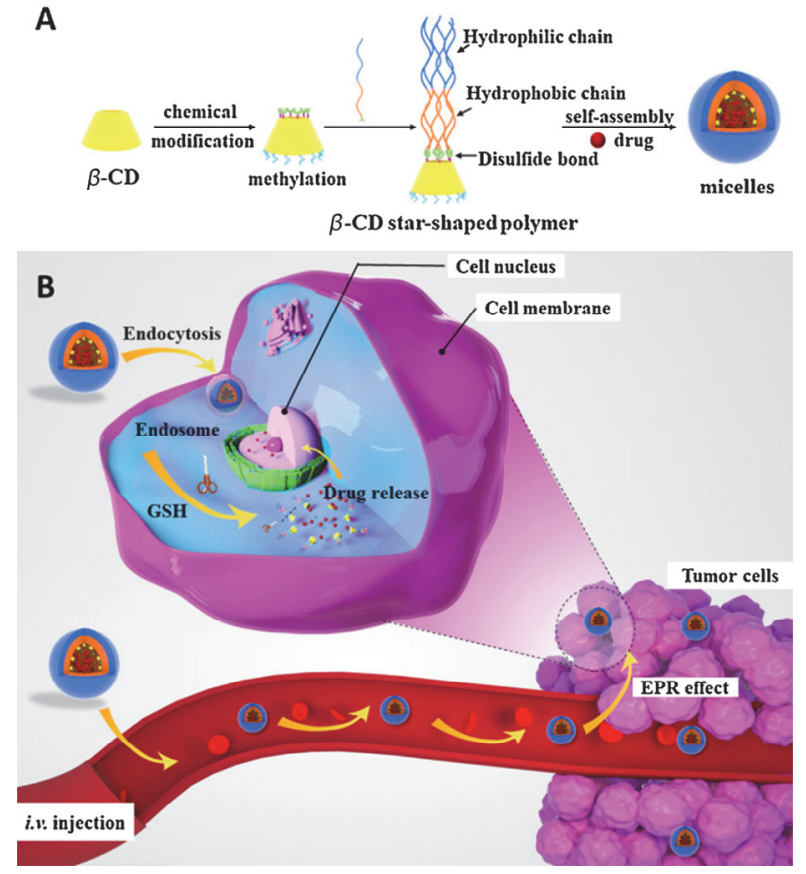

图 13 一系列环糊精星型聚合物的详细制备路线示意图及其可控载 药与释放应用 ${ }^{[84]}$

Figure 13 Detailed synthetic routes of a series of CD star-shaped polymer and their application for controlled drug delivery and release. Reprinted with permission from ref. ${ }^{[84]}$. Copyright $\subseteq 2017$ Elsevier B. V.
DOX 的负载率高达 $31.9 \%(w)$. 这些纳米胶束能够在肿 瘤区聚集并释放 DOX，表现出优异的靶向性.与此同 时，该胶束对其他部位的健康组织不造成明显影响，是 一种潜力巨大的肿瘤靶向载体.

\section{4 环糊精聚合的其他应用}

除了在生物医药领域有巨大的应用潜能, CDP 在环 境、隔热保温、催化、拓扑水凝胶和弹性体制备等方面 的应用也极具特色. 此处重点介绍交联 CDP 在环境、催 化、隔热领域中的应用以及 CD-PR 在拓扑水凝胶中的 应用。

CDP 在环境中的应用主要是污水处理，由于环糊 精分子与交联剂的协同作用，与传统的吸附剂，如活性 碳、离子交换树脂等相比较, 环糊精聚合物对极微量的 污染物也能起到有效吸附作用; 与纯环糊精相比, 环糊 精聚合物对亲水性污染物的吸附也更有效，例如高分子 量的染料分子和多环芳香烃化合物等. 最近, Crini 等 ${ }^{[13]}$ 以 EPI 交联环糊精聚合物为例，系统阐述了环糊精聚合 物的吸附机制(图 14), CDP 与吸附质的作用力主要有配 位、包结、物理作用、氢键、疏水作用、范德华力、静 电作用、离子相互作用和螯合作用等. 总体而言，与其 它吸附材料相比，环糊精聚合物与污染物的吸附可能同 
时存在化学吸附和物理吸附.

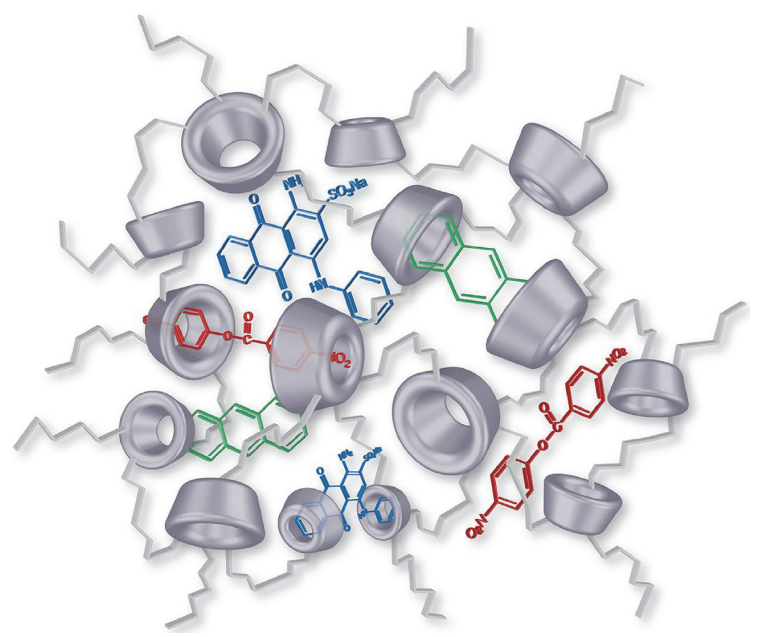

图 14 环糊精聚合物对有机小分子的吸附示意图 ${ }^{[13]}$

Figure 14 Schematic illustration of the absorption of small organic molecules by CDPs. Reprinted with permission from ref. ${ }^{[13]}$. Copyright@2017 Elsevier B. V.

CDP 在催化中的应用主要体现在其催化剂的负载 上, 如 Peinemann 等 ${ }^{[55]}$ 采用点击化学, 将叠氮修饰的环 糊精与对苯二乙炔交联聚合, 在 $60{ }^{\circ} \mathrm{C}$ 反应 $2 \mathrm{~d}$, 得到黄 色粘稠 CDP 沉淀物, 产量达 77\%. 值得一提的是, 该环 糊精聚合物交联部分可控, 所有环糊精的次级面完全保 留, 因此客体分子依然容易进入环糊精的空腔. 此外, 环糊精和交联剂都属于刚性基团, 得到的环糊精交联聚 合物具有优异的化学稳定性, 能够耐受水、DMF、THF、 丙酮、正己烷、乙醇和甲醇等. 以此 CDP 为模板, 利用 $\mathrm{CD}$ 的限域效应，制备得到尺寸小于 $1 \mathrm{~nm}$ 的金属 Pd 颗 粒. 这些环糊精聚合物负载的亚纳米颗粒具有非常高的 低温氢化 (Hydrogenation) 和铃木 - 宫浦偶合 (Suzuki-Miyaura coupling reaction)催化性能, 其中环糊 精对单体的吸附作用和 Pd 金属颗粒的超细尺寸起到一 定的协同作用. 此外, 以此环糊精聚合物为模板, 亦可 制备超细 $\mathrm{Ag} 、 \mathrm{Au} 、 \mathrm{Pt} 、 \mathrm{Rh}$ 等纳米粒子.

$\mathrm{CDP}$ 在隔热保温中的应用主要体现在 PR 气凝胶和 $\mathrm{CD}$ 气凝胶的成功制备上, 气凝胶是一类纳米多孔材料, 其孔隙率高达 99\%以上, 比表面积巨大, 热导率极低, 是一种密度极低、隔热性能极佳的固体材料 ${ }^{[86 ~ 91]}$. PR 气 凝胶于 2015 年被首次报道 ${ }^{[47]}$, 通过 $\alpha-\mathrm{CD}$ 与 $\mathrm{PEO}$ 在水 中自组装形成超分子水凝胶, 再通过超临界二氧化碳干 燥获得. 图 $15 \mathrm{~A}$ 展示了 $\mathrm{PR}$ 气凝胶的隔热性能, 分别制 备了 $\mathrm{PEG}$ 片 (热导率为 $0.3 \mathrm{~W} /(\mathrm{m} \cdot \mathrm{K})$ ), 聚苯乙烯 $(\mathrm{PS})$ 泡沫 片 (热导率约为 $0.1 \mathrm{~W} /(\mathrm{m} \cdot \mathrm{K})), \mathrm{PR}$ 气凝胶 $($ 热导率为 0.053 $\mathrm{W} /(\mathrm{m} \cdot \mathrm{K})$ ) 和氧化硅气凝胶 (热导率为 $0.0431 \mathrm{~W} /(\mathrm{m} \cdot \mathrm{K})$ ). 从图中可以看到, PEG 的温度很快上升并且熔化, PS 的 温度也随时间慢慢升高, 而 PR 和氧化硅气凝胶温度依 然维持较低温度, 表现出优异的隔热性能. 有意思的是, $\mathrm{PR}$ 气凝胶含有 $\mathrm{PEG}$ 结晶区域, 在 $40 \sim 50{ }^{\circ} \mathrm{C}$ 区间有熔
融吸热峰和冷却放热峰, 潜热可达 $71.8 \mathrm{~J} / \mathrm{g}$, 没有任何宏 观的体积变化和泄漏，因此又具有储能作用，使其将隔 热和储能有机融合于一体. 图 $15 \mathrm{~B}$ 是传统隔热材料的隔 热示意图，可以抵挡大部分热量从高温扩散至低温区 域, 反之亦然. 而 PR 气凝胶, 如图 $15 \mathrm{C}$ 所示, 其不仅能 够抵挡大部分热量从高温扩散至低温区域，同时能在材 料内部储存一定的能量, 当隔热材料两端温度变化时, PR 能够释放一定热量, 能够有效维持一端的温度不变. 此后, 研究人员相继制备出了温度刺激响应性聚轮烷气 凝胶 ${ }^{[48]}, \mathrm{CD}$ 气凝胶 ${ }^{[67,68]}$, 以及有机无机杂化气凝胶 等 ${ }^{[92 ~ 96]}$. 例如, Ito 等 ${ }^{[92]}$ 试图尝试直接将滑环胶交联制备 气凝胶, 但无法得到块体材料, 然而采用聚轮烷作为柔 性增韧基团修饰氧化硅表面, 制备得到环糊精氧化硅复 合气凝胶，该复合气凝胶具有高透明度，1 mm 厚的气凝 胶样品在 $550 \mathrm{~nm}$ 处的透光度能达到 $48.9 \%$. 密度控制在 $0.015 \sim 0.30 \mathrm{~g} / \mathrm{cm}^{3}$ 的范围，比表面积最高达到 $772 \mathrm{~m}^{2} / \mathrm{g}$. 此外, 该气凝胶的室温热导率仅为 $0.011 \mathrm{~W} /(\mathrm{m} \cdot \mathrm{K})$, 是一 种综合性能十分优异的复合氧化硅气凝胶. 此外, Xu 等 ${ }^{[95}$ 将 $\beta$-环糊精接枝到氧化石墨烯气凝胶上制备了环 糊精功能化的石墨烯气凝胶. 主要目的是利用环糊精对 抗体的选择性包覆, 实现对肿瘤标记物糖类抗原 15-3 (CA 15-3)的高精度免疫传感. 进一步, 他们用 $\beta$-环糊精 功能化嗍掺杂的氧化石墨烯气凝胶, 实现对二氯苯酚的 伏安传感检测 ${ }^{[96]}$.
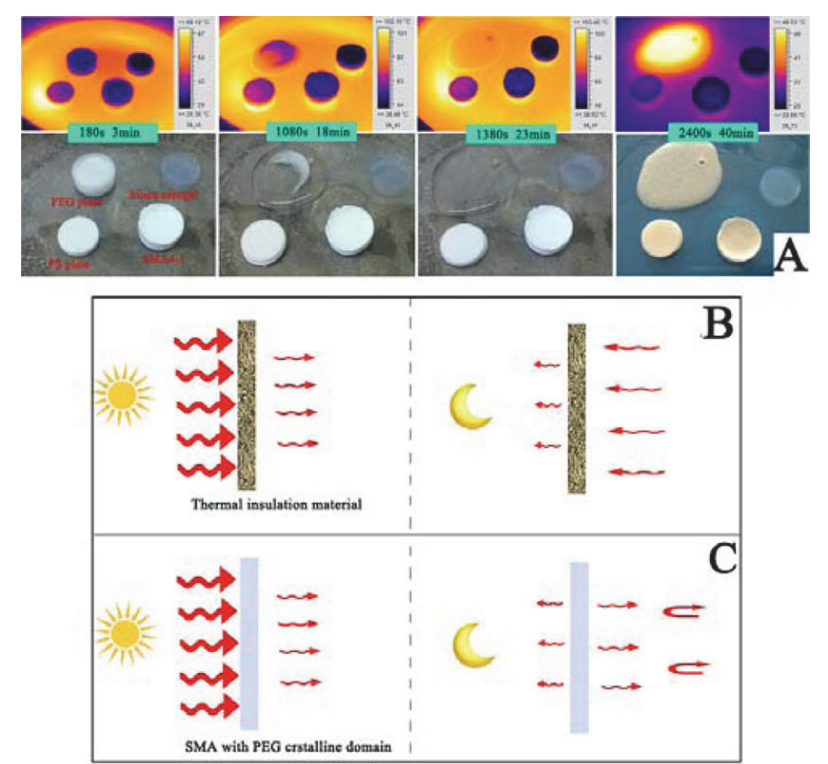

图 15 PR 气凝胶的隔热应用展示: (A) PEG 片、PS 片、氧化硅气凝 胶和 PR 气凝胶的实物和红外照片, 加热台温度 $100{ }^{\circ} \mathrm{C}$; (B)传统隔热 材料示意图; (C)隔热与储能一体化示意图 ${ }^{[47]}$

Figure 15 Application of PR aerogels for thermal insulation: (A) Infrared and digital photo images of PEG plate, PS plate, silica aerogel, PR aerogel heated under $100{ }^{\circ} \mathrm{C}$; (B) Illustration of conventional thermal insulation materials; and (C) Illustration of the combination of PCM and aeraogel for thermal insulation and heat preservation. Reprinted with permission from ref. ${ }^{[47]}$. Copyright $\bigcirc 2015$ American Chemical Society

除了生物医学应用, CD-PR 最独特的应用在于制备 拓扑凝胶, 又称滑环胶(slide-ring gel) ${ }^{[97 \sim 101]}$. 传统凝胶 
主要包括物理凝胶和化学凝胶 ${ }^{[102 ~ 104]}$, 物理凝胶主要由 非共价键形成的网络, 力学性能较差; 而化学凝胶由化 学键形成凝胶网络, 力学性能较强. 然而化学凝胶的交 联点固定, 因此在外力拉伸下容易产生应力集中而发生 断裂, 断裂伸长率较短.

而如图 16 所示 ${ }^{[97]}$, 将 PR 中的 CD 化学交联能够形 成一种全新的凝胶, 这种凝胶通过化学键交联, 形成 “ 8 ” 字型结构, 交联点可以在外力作用下滑动, 类似滑 轮一样, 因此被称为滑环胶. 滑环胶在外力拉伸下交联 点可以随着 $\mathrm{CD}$ 的运动而滑移, 能够消除局部应力, 使 得凝胶材料的拉伸长度大幅提高. 例如滑环胶的拉伸长 度可以达到初始长度的 24 倍, 体积可以在溶胀后增加 24000 倍. 当然, 实现 CD 的滑动除了外力, 还需两个因 素: (1) PR 中 CD 的数量少, 如此才具有足够的空间滑 动; (2)有一定的溶剂存在, 干态下聚合物堆积 CD 的滑 动受阻. 如通过小角 X-射线散射, Amemiya 等 ${ }^{[105]}$ 研究 了滑环胶中交联点的滑动特性. 研究发现在不良溶剂 中, 滑环胶聚集, 阻碍了滑环效应. 相反, 在良溶剂中, 聚合物链能够自由地穿过交联点从而表现出滑环效应, 在散射图中可以看到, 滑环胶在 $\mathrm{NaOH}$ 溶液中单轴拉伸 的衍射花样表现出各向同性, 而在不良溶剂中表现为非 正常的蝶形衍射花样. 这些结果表明在良溶剂中聚合物 能够在交联点上自由滑动, 而在不良溶剂中, 由于聚合 物不能自由滑动, 所以表现出与普通化学凝胶类似的特

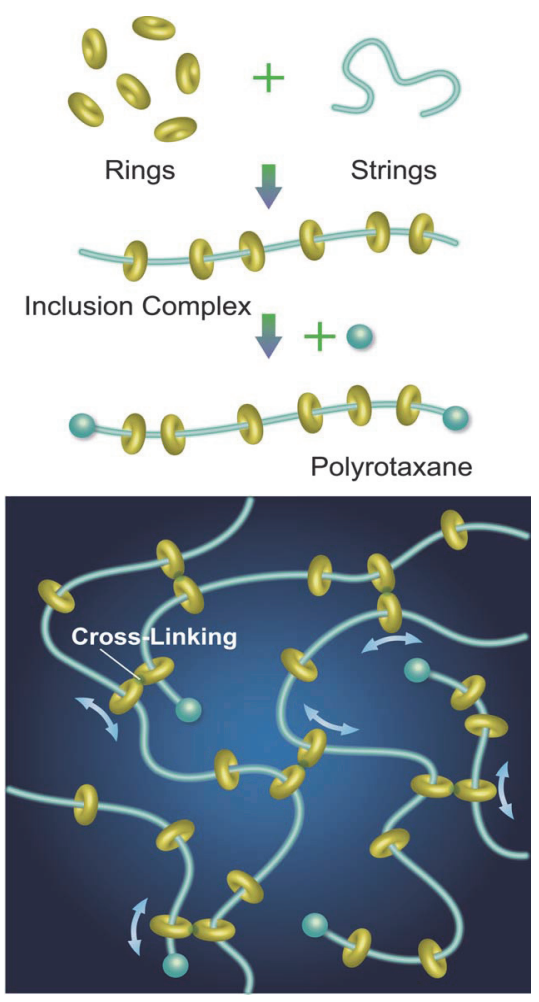

图 16 聚轮烷与滑环胶结构示意图 ${ }^{[97]}$

Figure 16 Schematic diagram of PR and slide-ring gel. Reprinted with permission from ref. ${ }^{[97]}$. Copyright $₫ 2014$ The authors and Journal of Applied Polymer Science Published by Wiley Periodicals, Inc.
点. 将 CD-PR 应用到滑环胶中，可制备拉伸性能优良， 具有自愈合性能的功能性材料, 极大地扩展了 CDP 的 应用领域。

\section{5 总结与展望}

环糊精聚合物集成了 CD 分子的特性与交联剂的性 质，同时额外获得了交联网络结构的性能，在环境领域 中具有独特的优势. 传统的 CDP 主要局限于 EPI 交联 物，虽然成本较低、制备技术成熟，但由于 EPI 的毒性， 其应用受到一定的局限. 近年来, 随着应用需求的扩 展、学科交叉的深入, 各类新型 CDP 不断涌现出来, 如 CDP 的种类从传统的交联 CDP, 到结构精细可控、能够 进一步自组装的 CD-PR、接枝型 CDP 以及 CD 星型聚 合物. 这些体系将 $\mathrm{CD}$ 的主体分子的包覆性能、优异的 生物相容性充分发挥出来, 在生物医药领域的应用取得 长足进展, 包括自洽抗体识别、肿瘤靶向输送、光控药 物释放、动脉继样斑块抑制等. 另一方面，传统 CDP 虽 然具有交联网络结构, 但干燥的样品比表面积低, 直接 影响其吸附能力, 近年来, 通过交联剂的设计, 得到比 表面积高达 $400 \mathrm{~m}^{2} / \mathrm{g}$ 的 CDP, 不仅增加了吸附能力, 而 且极大地提高了吸附速率. 随着 $\mathrm{CD}$ 气凝胶的出现, 又 将传统颗粒状的 CDP 制备成力学强度高达 $166 \mathrm{MPa}$ 的 块体材料, 实现了 CDP 从功能材料到高性能工程材料 的演变, 此外, $\mathrm{CD}$ 气凝胶不仅具有 $200 \mathrm{~m}^{2} / \mathrm{g}$ 以上的比表 面积, 而且密度低、具有丰富的多孔结构, 在同一材料 中实现亚纳米孔、微孔、介孔和大孔 4 种孔的共存, 且 表现出优异的隔热性能. 综上所述, 近年来, CDP 在结 构设计、性能优化、特殊应用等方面都取得重要进展，随 着学科的交叉而逐渐展现新的特色与生命力, 可以预 见, 随着 CDP 结构的进一步精细化设计和协同效应的 展示, CDP 在生物医药、节能环保、催化、智能响应材 料领域将获得重要应用.

\section{作者简介}

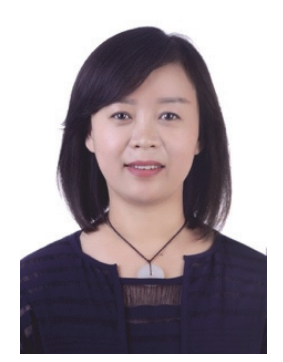

郄淑燕, 女, 主任医师、副教授, 首都医科大学附属北京 康复医院康复诊疗中心主任, 现任中华医学会物理医学与康 复学分会康复治疗学组副组长, 中国康复医学会康复机构管 理专委会常务委员, 中国康复医学会运动疗法专业委员会委 员、水中运动疗法学组副主委, 北京医师协会康复医学专科医 师分会常务理事, 石景山区重点学科学术带头人. 2018 年获 “北京优秀医师”。目前参与科技部重点课题 2 项, 主持省部级 
课题 1 项, 获省级科技进步奖 2 次. 主编著作 1 部, 主译著作 2 部, 副主编著作和教材 4 部, 以第一或通信作者发表 SCI 文 章 5 篇, 合作发表 SCI 文章 4 篇.

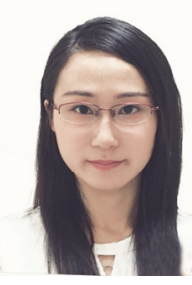

郝莹, 女, 副研究员. 2013 年毕业于苏州大学, 获高分子 化学与物理专业博士学位, 2014 年至 2017 年在美国 University of Delaware 材料科学与工程系从事博士后研究, 2017 年 8 月加 入中国科学院苏州纳米所, 任副研究员; 主要研究方向包括 高分子合成化学、功能生物材料以及干细胞定向分化研究; 主 持国家自然科学基金青年基金 1 项, 发表 SCI 论文 20 余篇, 获 中国发明专利授权 3 项.

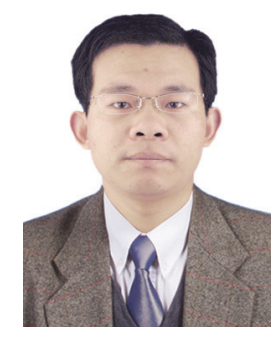

刘宗建, 男, 副研究员. 2013 年于北京理工大学生命学院 获应用化学博士学位, 2015 年于首都医科大学宣武医院神经 生物学博士后出站. 现担任首都医科大学附属北京康复医院 科研管理部主任、中国电子学会生命电子分会委员、中国老 年医学会脑血管分会青年委员. 主要研究方向为免疫调控和 炎症反应在脑卒中康复的作用机制研究, 主持国家自然科学 基金面上项目 1 项和北京市自然科学基金 1 项, 获教育部科技 进步奖 1 项. 以第一/通讯作者发表 SCI 收录论文 12 篇, EI 收 录论文 2 篇.

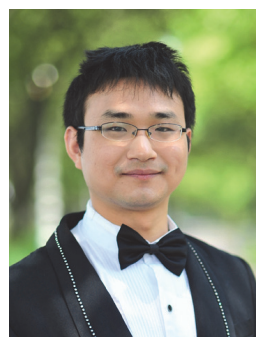

王锦, 男, 副研究员, 硕士生导师. 2012 年获北京理工大 学材料学博士学位, 2012 年至 2014 年分别于日本东京工业大 学和山形大学从事博士后研究, 2014 年回国加入中国科学院 苏州纳米所任副研究员. 主要研究方向为智能超分子水凝胶/ 气凝胶设计合成及其在生物、能源、环境中的应用, 迄今发表 SCI 论文 40 余篇, 申请发明专利 20 余项(已授权 8 项), 主持 国家自然科学基金 3 项、江苏省自然科学基金及苏州市科技 局等项目近 10 项, 作为主要研究人员参与国家重点研发计 划、中国科学院重点部署专项等数 10 项科研项目.

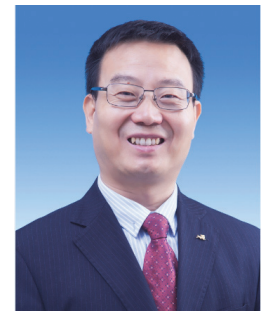

席家宁，男，教授，主任医师，硕士生导师，首都医科大 学附属北京康复医院、北京康复医学院院长, 首都医科大学康 复学系副主任, 康复医学与理疗学专业学科带头人. 成功带 领传统疗养院转型为学院型康复医院, 是现代康复医院建设 的引领者, “大康复, 强综合”康复医院运营管理模式的开拓者 和实践者. 创立了“以组织系统为基础, 以器官功能为核心”的 学科架构理论体系, 为中国康复医学事业发展做出了突出贡 献，荣获“首都劳动奖章”. 担任中国康复医疗机构联盟主席、 中国康复医学会康复机构管理专委会主任委员等. 主持多项 省部级及以上课题, 担任《中国康复科学杂志》主编、《中华 临床医师杂志》常务编审等，出版专业著作 6 部.

\section{References}

[1] Crini, G. Chem. Rev. 2014, 114, 10940.

[2] Yu, G.; Jie, K.; Huang, F. Chem. Rev. 2015, 115, 7240.

[3] Chen, Y.; Gui, X.; Duan, Z.; Zhu, L.; Xiang, Y.; Xia, D. Chin. J. Org. Chem. 2019, 39, 1284 (in Chinese). (陈雅琪, 桂金鍂, 段尊斌, 朱丽君, 项玉芝, 夏道宏, 有机化学, 2019, 39, 1284.)

[4] Shao, W.; Liu, X.; Wang, T.; Hu, X. Chin. J. Org. Chem. 2018, 38, 1107 (in Chinese). (邵为, 刘昕, 王婷婷, 胡晓玉, 有机化学, 2018, 38, 1107.)

[5] Liu, A.; Xiong, C.; Ma, X.; Ma, W.; Sun, R. Chin. J. Chem. 2019, $37,793$.

[6] Chen, X.; Chen, Y.; Liu, Y. Chin. J. Chem. 2018, 36, 526.

[7] Zhang, Y.; Chen, Y.; Li, J.; Liang, L.; Liu, Y. Acta Chim. Sinica 2018, 76, 622. (张依, 陈涌, 李晶晶, 梁璐, 刘育, 化学学报, 2018, 76, 622.)

[8] Ren, K.; He, J.; Zhang, M.; Wu, Y.; Ni, P. Acta Chim. Sinica 2015 73, 1038 (in Chinese). (任锴, 何金林, 张明祖, 吴一弦, 倪沛红, 化学学报, 2015, 73, 1038.)

[9] Li, Q.; Wang, J.; Ye, L.; Zhang, A.; Zhang, X.; Feng, Z. ChemNanoMat 2019, 5, 838 .

[10] Frieler, L.; Ho, T. M.; Anthony, A.; Hidefumi, Y.; Yago, A. J. E.; Bhandari, B. R. J. Food Sci. Technol. 2019, 56, 1519.

[11] Ho, T. M.; Bhandari, B. R. Powder Technol. 2015, 17, 585.

[12] Morin-Crini, N.; Crini, G. Prog. Polym. Sci. 2013, 38, 344.

[13] Morin-Crini, N.; Winterton, P.; Fourmentin, S.; Wilson, L. D.; Fenyvesi, E.; Crini, G. Prog. Polym. Sci. 2018, 78, 1.

[14] Manakker, F. V. D.; Vermonden, T.; Nostrum, C. F.; Hennink, W. E. Biomacromolecules 2009, 10, 3157.

[15] Solms, J.; Egli, R. H. Helv. Chim. Acta 1965, 48, 1225.

[16] Solms, J. US 3420788, 1969.

[17] Harada, A.; Li, J.; Kamachi, M. J. Am. Chem. Soc. 1994, 116, 3192.

[18] Xu, M.; Zhang, Y. Polym. Mater. Sci. Eng. 2010, 26, 162 (in Chinese). (徐美芸, 章永化, 高分子材料科学与工程, 2010, 26, 162.)

[19] Arisaka, Y.; Yui, N. J. Mater. Chem. B 2019, 7, 2123.

[20] Gao, P.; Wang, P.; Geng, X.; Ye, L.; Zhang, A.; Feng, Z. Acta Chim. Sinica 2013, 71, 347 (in Chinese). (高鹏, 王培境, 耿雪, 叶霖, 张 爱英, 冯增国, 化学学报, 2013, 71, 347.)

[21] Li, H.; Wang, J.; Ni, Y.; Zhou, Y.; Yan, D. Acta Chim. Sinica 2016, 74,415 (in Chinese). (李惠梅, 王洁, 倪云洲, 周永丰, 颜德岳, 化学学报, 2016, 74, 415.)

[22] Arunachalam, M.; Harry, W. G. Prog. Polym. Sci. 2014, 39, 1043.

[23] Hashidzume, A.; Yamaguchi, H.; Harada, A. Eur. J. Org. Chem. 2019, 21, 3344.

[24] Harada, A.; Hashidzume, A.; Yamaguchi, H.; Takashima, Y. Chem. Rev. 2009, 109, 5974.

[25] Okada, M.; Kamachi, M.; Harada, A. Macromolecules 1999, 32, 7202.

[26] Harada, A.; Kawaguchi, Y.; Nishiyama, T.; Kamachi, M. Macromol. 
Rapid. Commun. 1997, 18, 535.

[27] Rusa, C. C.; Tonelli, A. E. Macromolecules 2000, 33, 5321.

[28] Harada, A.; Suzuki, S.; Okada, M.; Kamachi, M. Macromolecules 1996, 29, 5611.

[29] Okumura, H.; Kawaguchi, Y.; Harada, A. Macromolecules 2001, 34, 6338.

[30] Loethen, S.; Kim, J. M.; Thompson, D. H. Polym. Rev. 2007, 47, 383.

[31] Wang, P. J.; Wang, J.; Ye, L.; Zhang, A.; Feng, Z. G. Polymer 2012, $53,2361$.

[32] Wang, J.; Li, S.; Ye, L.; Zhang, A.; Feng, Z. G. Macromol. Rapid Commun. 2012, 33, 1143.

[33] Kato, K.; Kamotsu, H.; Ito, K. Macromolecules 2010, 43, 8799.

[34] Yu, S.; Zhang, Y.; Wang, X.; Zhen, X.; Zhang, Z.; Wu, W.; Jiang, X. Angew. Chem., Int. Ed. 2013, 52, 7272.

[35] Zhang, X. W.; Zhu, X. Q.; Tong, X. M.; Ye, L.; Zhang, A.; Feng, Z. G. J. Polym. Sci. Part A: Polym. Chem. 2008, 46, 5283.

[36] Ren, L. X.; Ke, F. Y.; Chen, Y. M.; Liang, D.; Huang, J. Macromolecules 2008, 41, 5295.

[37] Dai, X. H.; Dong, C. M.; Yan, D. Y. J. Phys. Chem. B 2008, 112, 3644.

[38] Wang, J.; Gao, P.; Ye, L.; Zhang, A.; Feng, Z. G. J. Phys. Chem. B 2010, 114, 5342.

[39] Wang, J.; Ye, L.; Zhang, A.; Feng, Z. G. J. Mater. Chem. 2011, 21, 3243 .

[40] Wang, J.; Gao, P.; Wang, P. J.; Ye, L.; Zhang, A.; Feng, Z. G. Polymer 2011, 52, 374.

[41] Wang, J.; Gao, P.; Ye, L.; Zhang, A.; Feng, Z. G. Polym. Chem. 2011, 2, 931.

[42] Wang, J.; Wang, P. J.; Ye, L.; Zhang, A.; Feng, Z. G. Polymer 2011, 52,5362 .

[43] Wang, J.; Gao, P.; Jiang, L.; Ye, L.; Zhang, A.; Feng, Z. G. Polymer 2012, 53, 2864.

[44] Duan, N.; Lu, H.; Ye, L.; Zhang, A.; Feng, Z. G. J. Phys. Chem. B 2019, 123, 5004 .

[45] Kong, T.; Ye, L.; Zhang, A.; Feng, Z. G.; Langmuir 2018, 34, 14076.

[46] Gao, M.; Lu, H.; Song, R. H.; Ye, L.; Zhang, A.; Feng, Z. G. Polym. Chem. 2020, 11, 653 .

[47] Wang, J.; Zhang, X. ACS Nano 2015, 9, 11389.

[48] Wang, J.; Du, R.; Zhang, X. ACS Appl. Mater. Interfaces 2018, 10, 1468 .

[49] Uenuma, S.; Maeda, R.; Yokayama, H.; Ito, K. Chem. Commun. 2019, 55, 4158 .

[50] Uenuma, S.; Maeda, R.; Yokoyama, H.; Ito, K. Macromolecules 2019, 52, 3881.

[51] Arisaka, Y.; Yui, N. Macromol. Rapid Commun. 2019, 40, 1900323.

[52] Demirci, S.; Kinali-Demirci, S.; Jiang, S. Chem. Commun. 2017, 53, 3713.

[53] Zhang, L.; Zhao, J.; Wang, Y. Acta Chim. Sinica 2015, 73, 1182 (in Chinese). (张丽芳, 赵杰, 王勇, 化学学报, 2015, 73, 1182.)

[54] Lucio, D.; Martinez-Oharriz, M. C.; Gu, Z.; He, Y.; Aranaz, P.; Vizmanos, J. L.; Irache, J. M. Int. J. Pharm. 2018, 547, 97.

[55] Azmeera, V.; Tungala, K.; Adhikary, P.; Kumar, K.; Krishnamoorthi, S. Int. J. Biol. Macromol. 2017, 104, 1204.

[56] Li, L.; Guo, X.; Wang, J.; Liu, P.; Prud'homme, R. K.; May, B. L.; Lincoln, S. F. Macromolecules 2008, 41, 8677.

[57] Wang, J.; Guo, Z.; Xiong, J.; Wu, D.; Li, S.; Tao, Y.; Qin, Y.; Kong, Y. Int. J. Biol. Macromol. 2019, 125, 941.

[58] Shen, Y.; Niu, L.; Yu, Z.; Wang, M.; Shang, Z.; Yang, Y. Appl. Surf. Sci. 2018, 444, 42.

[59] Pooresmaeil, M.; Namazi, H. Colloids Surf. B: Biointer. 2018, 172, 17.

[60] Zhang, B.; Yu, Q.; Zhang, Y. M.; Liu, Y. Chem. Commun. 2019, 55, 12200.

[61] Bai, L.; Yan, H.; Bai, T.; Feng, Y.; Zhao, Y.; Ji, Y.; Feng, W.; Lu, T.; Nie, Y. Biomacromolecules 2019, 20, 4230.

[62] Baimani, N.; Azar, P. A.; Husain, S. W.; Panahi, H. A.; Mehramizi, A. J. Chromatogr. A 2018, 1571, 38.

[63] Helal, A. S.; Mazario, E.; Mayoral, A.; Decorse, P.; Losno, R.; Lion, C.; Ammar, S.; Hemadi, M. Environ. Sci. Nano 2018, 5, 158.

[64] Hong, S.; Li, Z.; Li, C.; Dong, C.; Shuang, S. Appl. Surface Sci. 2018, 427, 1189.

[65] Alsbaiee, A.; Smith, B. J.; Xiao, L.; Ling, Y.; Helbling, D. E.; Dichtel, W. R. Nature 2016, 529, 190.
[66] Xu, G.; Xie, X.; Qin, L.; Hu, X.; Zhang, D.; Xu, J.; Li, D.; Ji, X.; Huang, Y.; Tu, Y.; Jiang, L.; Wei, D. Green Chem. 2019, $21,6062$.

[67] Wang, J.; Wang, X.; Zhang, X. J. Mater. Chem. A 2017, 5, 4308.

[68] Lenohardt, E. E.; Meador, M. A. B.; Wooley, K. L. Chem. Mater. 2018, 30, 6226 .

[69] Mizuno, S.; Asoh, T. A.; Takashima, Y.; Harada, A.; Uyama, H. Polym. Degrad. Stability 2019, 160, 136.

[70] Kretschmann, O.; Choi, S. W.; Miyauchi, M.; Tomatsu, I.; Harada, A.; Ritter, H. Angew. Chem. Int. Ed. 2006, 45, 4361.

[71] Cheng, H.; Fan, X.; Wu, C.; Wang, X.; Wang, L. J.; Loh, X. J.; Li, Z.; Wu, Y. L. Macromol. Rapid Commun. 2019, 40, 1800207.

[72] Shukula, A.; Singh, A. P.; Ray, B.; Aswal, V.; Kar, A. G.; Maiti, P. J. Colloid Interface Sci. 2019, 534, 215.

[73] Cocq, A.; Rousseau, C.; Bricout, H.; Oliva, E.; Bonnet, V.; Djedaini-Pilard, F.; Monflier, E.; Tilloy, S. Eur. J. Org. Chem. 2019, 4863.

[74] Furlan, A. L.; Buchoux, S.; Miao, Y.; Banchet, V.; Leteve, M.; Lambertyn, V.; Michel, J.; Sarazin, C.; Bonnet, V. New J. Chem. 2018 42, 20171.

[75] Arslan, M.; Sanyal, R.; Sanyal, A. Polym. Chem. 2020, 11, 615.

[76] Seo, J. H.; Kakinoki, S.; Inoue, Y.; Yamaoka, T.; Ishihara, K.; Yui, N. J. Am. Chem. Soc. 2013, 135, 5513.

[77] Zhang, Y.; Zhou, Q.; Jia, S.; Lin, K.; Fan, G.; Yuan, J.; Yu, S.; Shi, J. ACS Appl. Mater. Interfaces 2019, 11, 46427.

[78] Rajendran, A. K.; Arisaka, Y.; Iseki, S.; Yui, N. ACS Biomater. Sci. Eng. 2019, 5, 5652.

[79] Srinivasachari, S.; Fichter, K. M.; Reineke, T. M. J. Am. Chem. Soc. 2008, 130, 4618.

[80] Li, J.; Yang, C.; Li, H.; Wang, X.; Goh, S. H.; Ding, J. L.; Wang, D. Y.; Leong, K. W. Adv. Mater. 2006, 18, 2969.

[81] Zhang, J.; Zhang, L.; Li, S.; Yin, C.; Li, C.; Wu, W.; Jiang, X. ACS Biomater. Sci. Eng. 2018, 4, 1963.

[82] Zhang, Y.; Zhang, Z.; Chen, W.; Li, C.; Wu, W.; Jiang, X. Acta Polym. Sinica 2017, 48, 306 (in Chinese). (张亚军, 张正奎, 陈伟 芝, 李成, 武伟, 蒋锡群, 高分子学报, 2017, 48, 306.)

[83] Kim, H.; Han, J.; Park, J. H. J. Control. Release 2020, 319, 77.

[84] Li, X.; Liu, H.; Li, J.; Deng, Z.; Li, L.; Liu, J.; Yuan, J.; Gao, P.; Yang, Y.; Zhong, S. Colloids Surf. B: Biointer. 2019, 183, 110425.

[85] Huang, T.; Sheng, G.; Manchanda, P.; Emwas, A. H.; Lai, Z.; Nunes, S. P.; Peinemann, K. V. Sci. Adv. 2019, 5, eaax6976.

[86] Pierre, A. C.; Pajonk, G. M. Chem. Rev. 2002, 102, 4243.

[87] Wang, J.; Wei, Y.; He, W.; Zhang, X. RSC Adv. 2014, 4, 51146.

[88] Wang, J.; Zhang, Y.; Wei, Y.; Zhang, X. Micropor. Mesopor. Mater. 2015, 218, 192.

[89] Wang, J.; Zhang, Y.; Zhang, X. J. Mater. Chem. A 2016, 4, 11408.

[90] Li, X.; Wang, J.; Zhao, Y.; Zhang, X. ACS Appl. Mater. Interfaces 2018, 10, 16901.

[91] Liu, R.; Wang, J.; Du, Y.; Liao, J.; Zhang, X. J. Solid State Chem. 2019, 279, 120971.

[92] Jiang, L.; Kato, K.; Mayumi, K.; Yokoyama, H.; Ito, K. ACS Macro Lett. 2017, 6, 281.

[93] Matias, T.; Marques, J.; Conceicao, F.; Maleki, H.; Quina, M. J.; Gando-Ferreira, L.; Valente, A. J. M.; Portugal, A.; Duraes, L. J. Sol-Gel Sci. Technol. 2017, 84, 409.

[94] Zhou, K.; Li, Y.; Li, Q.; Du, Q.; Wang, D.; Sui, K.; Wang, C.; Li, H.; Xia, Y. J. Polym. Environ. 2018, 26, 3362.

[95] Jia, H.; Tian, Q.; Xu, J.; Lu, L.; Ma, X.; Yu, Y. Microchim. Acta 2018, $185,517$.

[96] Xie, Y.; Tu, X.; Ma, X.; Fang, Q.; Lu, L.; Yu, Y.; Liu, G.; Liu, C. Nanotechnology 2019, 30, 185502.

[97] Noda, Y.; Hayashi, Y.; Ito, K. J. Appl. Polym. Sci. 2014, 131, 40509.

[98] Li, S.; Wang, J.; Gao, P.; Ye, L.; Zhang, A.; Feng, Z. G. Sci. China Chem. 2012, 55, 1115.

[99] Li, S.; Wang, J.; Jiang, L.; Ye, L.; Zhang, A.; Feng, Z. G. Chin. J. Chem. 2012, 30, 2453.

[100] Okumura, Y.; Ito, K. Adv. Mater. 2001, 13, 48.

[101] Araki, J.; Ito, K. Soft Matter 2007, 3, 1456.

[102] Voorhaar, L.; Hoogenboom, R. Chem. Soc. Rev. 2016, 45, 4013.

[103] Tan, M.; Wang, J.; Song, W.; Fang, J.; Zhang, X. J. Mater. Chem. A 2019, 7, 1244.

[104] Buwalda, S. J.; Boere, K. W. M.; Dijkstra, P. J.; Feijen, J.; Vermonden, T.; Hennink, W. E. J. Control. Release 2014, 190, 254.

[105] Shinohaea, Y.; Kayashima, K.; Okumura, Y.; Zhao, C.; Ito, K.; Amemiya, Y. Macromolecules 2006, 39, 7386. 